COMPUTER CONTROLLED MHD POWER CONSOLIDATION AND PULSE GENERATION SYSTEM

Final Technical Progress Report for the Perlod October 1987-August 1990
By
R. Johnson
K. Marcotte
M. Donnelly

Work Performed Under Contract No. AC22-87PC79680

For

U.S. Department of Energy

Plttsburgh Energy Technology Center

Pittsburgh, Pennsylvania

By

Montana State University

Bozeman, Montana 


\section{DISCLAIMER}

This report was prepared as an account of work sponsored by an agency of the United States Government. Neither the United States Government nor any agency thereor. nor any of their employees, makes any warranty, express or implied. or assumes any legal liability or responsibility for the accuracy, completeness, or usefulness of any information, apparatus, praduct, or process disclosed. ur represents that its use would not infringe privately owned rights. Reference herein to any specific commercial product, process. or service by trade name. trademark. manufacturer. or otherwise does not necessarily constitute or imply its endorsement. recommendation. or favoring by the Unit ed States Government or any agency thereof. The views and opinions of authors ex. pressed herein do not necessarily state or reflect those of the United States Government or any agency thereor.

This report has been reproduced directly from the best available copy.

A vailable to DOE and DOE contractors from the Office of Scientific and Technical Information, P.O. Box 62, Oak Ridge, TN 37831; prices available from (615) 576.8401 , FTS 626.8401.

A vailable to the public from the National Technical Information Service, U.S. Department of Commerce, 5285 Port Royal Rd., Springfield, VA 22161. 
Computer Controlled MHD Power Consolidation and Pulse Generation System

Final Technical Progress Report

Oct. 1987 - Aug. 1990

by

R. Johnson, K. Marcotte, and M. Donnelly Department of Electrical Engineering Montana State University

Bozeman, MT 59717-0007 (406)-994-4271

ERL REPORT 290666-3

Contract: DE-AC22-87PC79680

A Research Project Sponsored by The United States Department of Energy MHD/SDI Division

Pittsburgh Energy Technology Center

P.O. Box 10940-MS900-33

Pittsburgh, PA 15236 


\subsection{TABLE OF CONTENTS}

Section

1.0

1.1

1.2

2.0

3.0

3.1

3.2

3.2 .1

3.2 .2

3.3

3.3.1

3.3.1.1

3.3.1.2

3.3.1.3

3.4

3.5

3.5.1

3.5 .2

3.5 .3

3.5 .4

3.5 .5

3.5 .6

3.5 .7

3.5 .8

3.5 .9

3.5 .10

3.5 .11

3.5 .12

3.5 .13

3.5 .14

3.6

3.5 .15

3.6.1

3.6 .2

TITLE PAGE

TABLE OF CONTENTS

LIST OF FIGURES

GLOSSARY OF TERMS

INTRODUCTION

WORK PROGRESS

PASC Concept Evolution and Comparisons: inception Faraday, Feasibility Demonstration, Inductive and Capacitive Consolidation, and single terminal diagonal.

Preliminary computer simulation results from

applying the PASC technology to the diagonal generator connection.

Modeling the PASC Process Using EMTP

Discussion of Results 15

Feasibility Demonstration Test Results Using Low 18

Power Semiconductors

Summary of PASC Feasibility Demonstration 19

Low-power Test Results

Computer controls system operation

Versatility of waveform generation

Efficiency requirements

19

Feasibility Demonstration Test results obtained $\quad 20$

using intermediate-level power semiconductors.

Description of some preliminary harmonic sup-

pression control waveform algorithms and a new

data acquisition system and control system.

Introduction

The Internal Model Principle $\quad 24$

The Continuous-Time System $\quad 26$

Discretization of the System $\quad 26$

Simulations $\quad 29$

Conclusions $\quad 32$

The Harris Design Contest Controller $\quad 32$

The Digital Controller $\quad 33$

The Continuous Fourier Transform $\quad 34$

Hardware Interface With The Existing System

Software Implementation $\quad 37$

The Sampling Period and Controller Interrupts 38

$\begin{array}{ll}\text { The CFT } & 39\end{array}$

Memory Allocation $\quad 40$

Conclusions and Discussion $\quad 41$

Final calculation results for a space-deployed $\quad 41$

minimum power-to-weight ratio PASC system at a

100 inî̀i level.

Basic Assumptions Used In The Power-to weight 42

Ratio Calculation

Design of the PASC Transformer 
3.6 .3

3.6 .4

4.0

5.0

6.0
Final Design Transformer Loss Calculations

43

Switching Losses and Final Power-to-weight Ratio

44

Results

FINAL CONCLUSIONS AND RECOMMENDA-

45

TIONS

REFERENCES

APPENDIX A: Harris RTX2000 Code Listings
46

47 


\section{LIST OF FIGURES}

Figure Figure Title $\quad$ Page

Number

PASC Faraday-connectedConsolidation/Inversion System For 8 Sources $\quad 8$

Early Conceptual PASC System Diagram $\quad 9$

3 Optical Isolation Computer-to-Switching Interface 10

4 Adjacent Electrode Parallel Loading to Obtain Bipolar consolidation and 10 three phase Inversion

5 DEC Microvax II Master Controller with KX.-11 SBC Slave Switching Control Sequencer

6 Four Core, 16 Primary, Parallel Secondary PASC Transformer 12

7 Two-Primary Winding CI Transformers With Series Connected Secondaries 13

8 PASC Four-pulse CI System For A Diagonally Connected MHD Generator 14

9 Diagonal Output Voltage for Rsource $=10 \mathrm{ohms}$, Rload $=1 \mathrm{ohm} \quad 16$

10 Diagonal Configuration. Rsource $=1 \mathrm{ohm}$, Rload $=100 \mathrm{ohms} \quad 17$

11 Diagonal Configuration. Rsource $=1 \mathrm{ohm}$, Rload $=10 \mathrm{ohms} \quad 17$

12 Diagonal Configuration. Rsource $=1 \mathrm{ohm}$, Rload $=1 \mathrm{ohm} \quad 17$

13 Load voltage for $2.5 \mathrm{ohm}$ source impedance and $100 \mathrm{ohm}$ load impedance for 20 step-down transformer connection.

14 Load voltage for $2.5 \mathrm{ohm}$ source impedance and $10 \mathrm{ohm}$ load impedance for 21 step-down transformer connection.

15 Load voitage for $2.5 \mathrm{ohm}$ source impedance and $100 \mathrm{ohm}$ load impedance for 21 step-up transformer connection.

16 Load voltage for $2.5 \mathrm{ohm}$ source impedance and $10 \mathrm{ohm}$ load impedance for step-up transformer connection.

17 Load voltage for $10 \mathrm{ohm}$ source impedance and $100 \mathrm{ohm}$ load impedance for step-down transformer cornection.

18 Load voltage for $10 \mathrm{ohm}$ source impedance and $100 \mathrm{ohm}$ load impedance for step-up transformer connection.

19 Load voltage for $10 \mathrm{ohm}$ source impedance and $10 \mathrm{ohm}$ load impedance for step-down transformer connection.

20 Load voltage for $10 \mathrm{ohm}$ source impedance and $10 \mathrm{ohm}$ load impedance for step-up transformer connection.

21 Internal Model of Sinusoidal Reference 25

22 Transformer model for simulation 25

23 Closed-loop System 26

24 The Discrete-Time System 27

25 Model and Controller Test 27 
26 Root Locus on $k_{1}$

27 Root Locus onk 29

$28 \quad \mathrm{R}=100, k_{1}=1, k_{2}=1$

$29 \mathrm{R}=100, k_{1}=1, k_{2}=10$

30 Spectrum of Figure 28

$31 \mathrm{R}=1, k_{1}=1, k_{2}=0.1 \quad 30$

32 Spectrum of Figure 30

$33 \mathrm{~F}=600 \mathrm{~Hz}, R=10, k_{1}=1, k_{2}=1$

$34 \quad$ Spectrum of Figure 33

35 The closed-loop system in continuous time. 34

36 Block diagram of closed-loop system. 34

$37 \quad$ Chip layout on RTXEB.

$38 \quad$ Logic diagram for Mux. 36

$39 \quad$ A/D pinout and external clock. $\quad 37$

$40 \quad$ CFT and Interrupt flowcharts. 39

$41 \quad$ Mernory map. $\quad 40$ 


\section{GLOSSARY OF TERMS}

Term or Abreviation ac

\section{A/D}

ASIC bus

CFT

consolidation

dc

diagonal mode

EMTP

EPRI

Faraday mode

GTO

$\mathrm{Hz}$

inversion

I/O

MHD

Mosfet

Mux

MWe

PASC

RTX

RTXEB

SBC

snubber

SPICE

SRAM

Tesla

Thevenin equivalent

thyristor

Tri-state

UART
Description

alternating current

Analog-to-Digital

Application specific integrated circuit bus

continuous Fourier Transform

the consolidation of multiple-dc sources into a single source direct current

MHD connection employing both Hall and Faraday fields

Electro-Magnetics-Transient-Program (EPRI)

Electric Power Research Institute, Palo Alto, CA

MHD connection employing only the Faraday electric field

Gate-Tum-Off thyristor switch

Hertz, frequency in cycles/second

The process of generating ac waveforms from dc sources

Input-output

Magnetohydrodynamic

Metal oxide semiconductor field effect transistor

Multiplexor, many-to-one consolidation

Megawatts electric

Pulse-Amplitude-Synthesis-and-Control

Real-Time-Express (Harris Corporation trademark)

RTX evaluation board

single board computer

a sub-circuit to allow for continuity of current flow through a switched inductor

Simulation Program for Integrated Circuit Evaluation

static random access memory

Measure of magnetic flux ciensity $=10^{4}$ Maxwells

Network theorem equivalent terminal voltage and impedance representation

non-reciprocal threshhold voltage circuit element

solid state device providing latching of temporary bus information signals

Universal Asynchronous Receive and Transmit circuit 


\author{
Computer Controlled MHD Power Consolidation \\ and Pulse-Generation System \\ Final Technical Progress Report \\ Oct. 1989 - Aug. 1990
}

\title{
2.0 INTRODUCTION
}

The major goal of this research project is to establish the feasibility of a power conversion technology which will permit the direct synthesis of computer programmable pulse power. Feasibility has been established in this project by demonstration of direct synthesis of commercial frequency power by means of computer control. The power input to the conversion system is assumed to be a Faraday connected MHD generator which may be viewed as a multi-terminal dc source and is simulated for the purposes of this demonstration by a set of dc power supplies. This consolidation/inversion (CI), process will be referred to subsequently as Pulse Amplitude Synthesis and Control (PASC).

A secondary goal is to deliver a controller subsystem consisting of a computer, software, and computer interface board which can serve as one of the building blocks for a possible phase II prototype system.

\subsection{WORK PROGRESS}

This report period work summarizes the accomplishments and covers the high points of the two year project. Progress is reported by reference to the original contract, and includes discussions on the following areas:

Comparison of the original PASC concept as proposed at the project inception, the Feasibility Demonstration system design, and the final resulting concept at the end of the project.

Preliminary computer simulation results from applying the PASC technology to the diagonal generator connection.

Feasibility Demonstration Test results using low-level power semiconductors.

Feasibility Demonstration Test results obtained using intermediate-level power semiconductors.

Description of some preliminary harmonic suppresion control waveform algorithms and a new data acquisition system and control system.

Final calculation results for a space-deployed minimum power-to-weight ratio PASC system at a $100 \mathrm{MW}=$ level.

3.1 PASC Concept Evolution and Comparisons: Inception, Feasibility Demonstration, Inductive and Capacitive Consolidation, and Single Terminal.

The original PASC concept at contract inception is shown in Figure 1 in block diagram form for a Faraday connected MHD generator. 


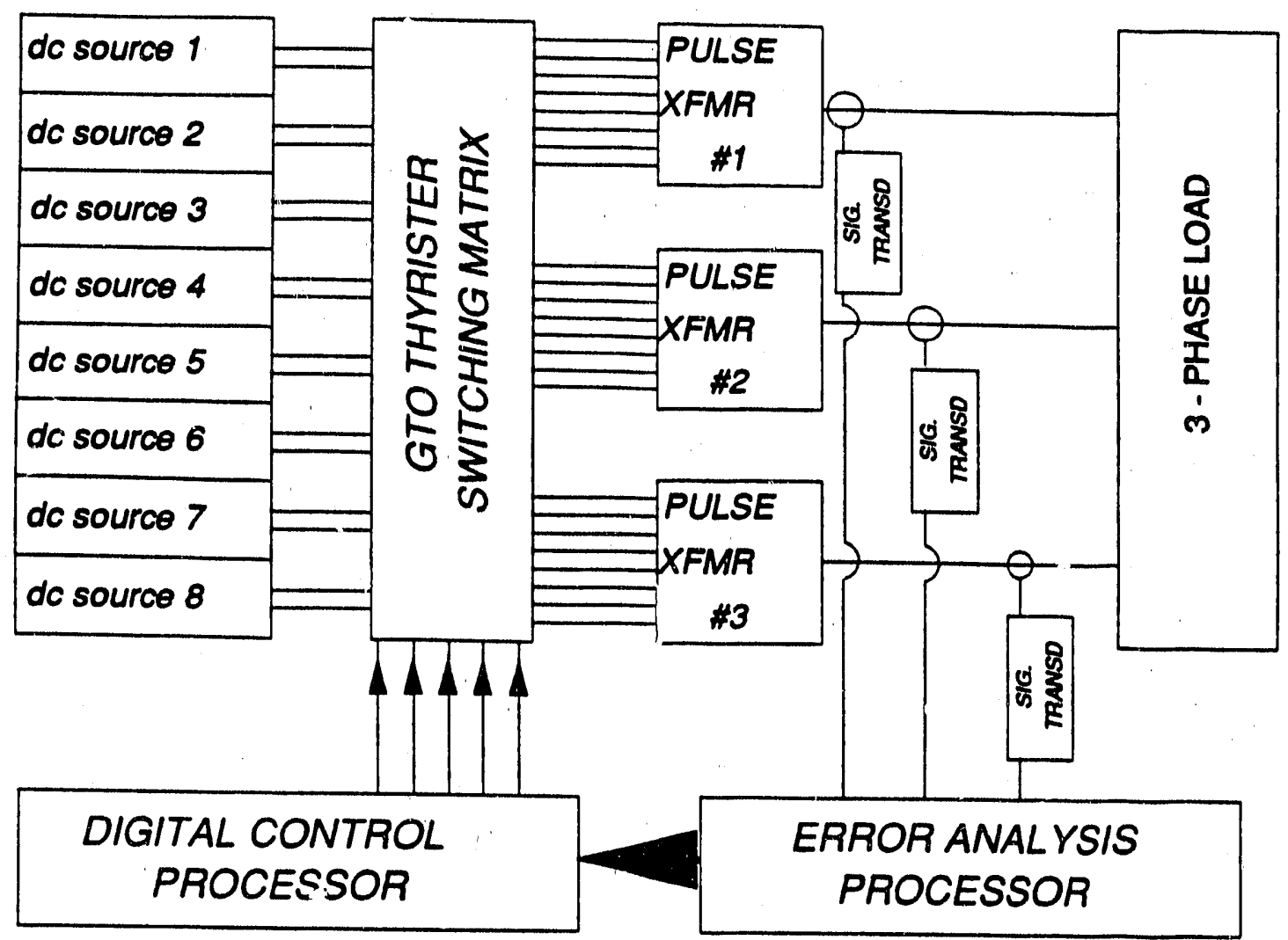

Figure 1. PASC Faraday-connecied Consolidation/Inversion System For 8 Sources

A quite complex transformer was originally envisioned assuming that only a single transformer was going to be used for consolidating all of the Faraday individual de sources along with generation of a single phase alternating current waveform. Although consolidation and superposition using the PASC concept can be implemented either magnetically using inductors or capacitively with capacitors, inductors were chosen for the Feasibiltiy Demonstration design because they are generally lower in cost, more rugged, and provide significant voltage isolation between windings. Negative aspects of transformers include their weight, nonlinearities, requirement that no average dc signals be present, and somewhat higher losses compared to capacitors per unit of energy storage. At the beginning it was not realized how important exact magnetic symmetry was for proper balancing of power output over the transformer(s) as is indicated in Figure 2 which shows asymmetry of magnetic circuit coupling depending on the location of the primary windings. Subsequent experimental results along with computer analysis show that symmetry of coupling coefficients, losses, etc., are quite important for achieving minimal loss performance, symmetry with respect to saturation effects, and wide frequency bandwidth of operation which are neccesary for pulse applications. Other changes from the original inception diagram shown in Figure 2 included: 


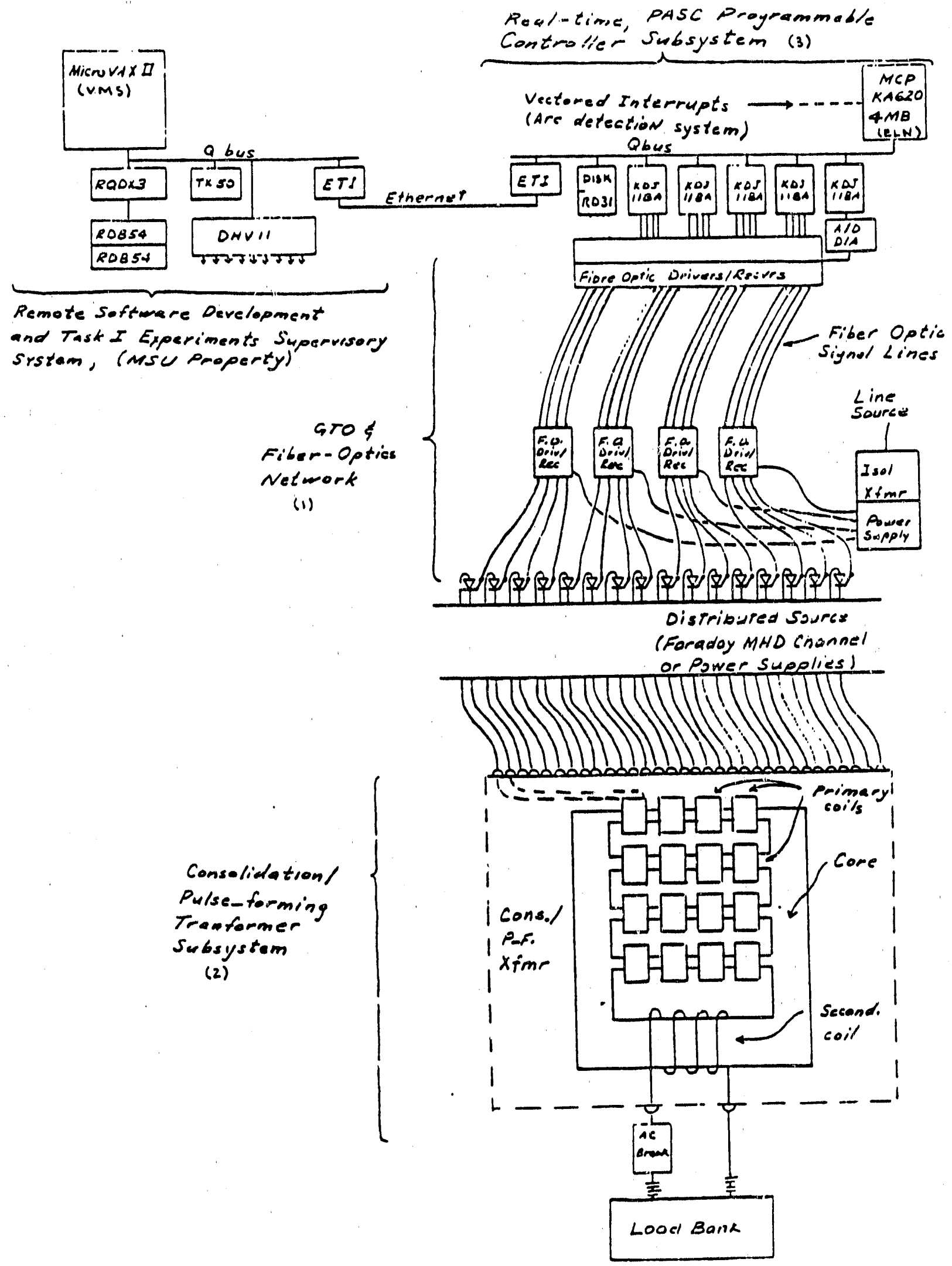

Figure 2 Early Conceptual PASC System Diagram 
a. The usage of optically isolated computer level switching transistors rather than fiber optics circuits to achieve isolation of the computer control circuitry from the heavy current test circuity. In an actual system fiber optics would be used because the power voltages would exceed tens of kilovolts whereas in the Feasibility Demonstration system all voltages were less than one kilovolt. Such an isolation system is indicated in Figure 3.

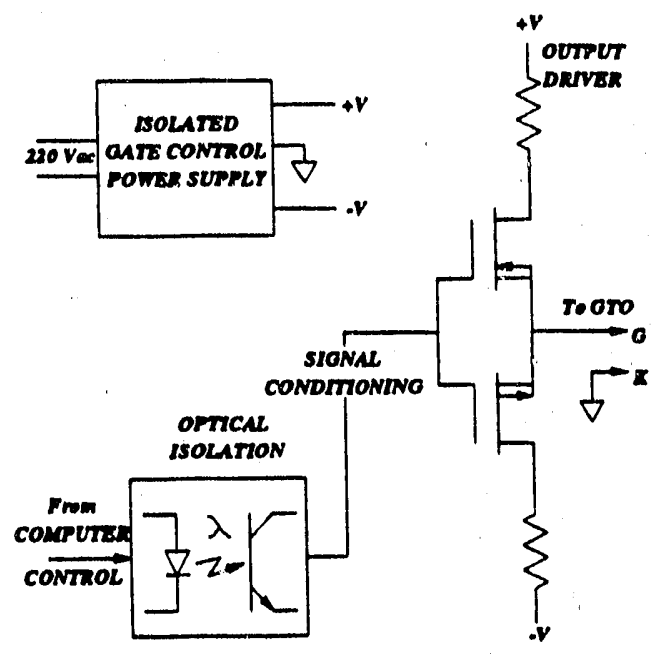

Figure 3. Optical Isolation Computer-to-Switching Interface

b. Because of cost and time considerations it was decided at the time of detailed design of the Feasibility Demonstration system to only implement a single-phase rather than a three-phase inverter system. A three phase inverter system involves no additional design or demonstration unknowns relative to a single-phase system: it consists of three, single-phase inverters connected in parallel across the sources as shown in Figure 4.

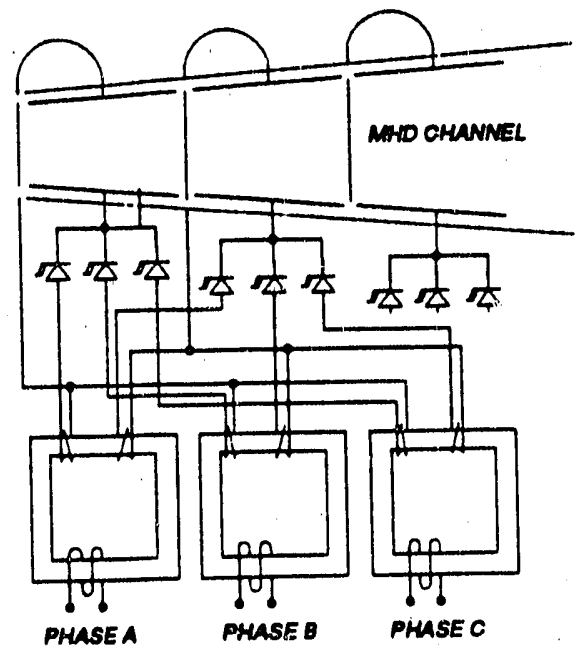

Figure 4.Adjacent Electrode Parallel Loading to Obtain Bipolar consolidation and three-phase Inversion 
c. Rather than use a remote Microvax II processor to develop the software for PASC GTO switching control with rittwork down-line loading to a dedicated control Microvax II running the ELAN real-time operating system, the control Micro ax II was purchased with the VMS operating system. Software was then developed directly on the Microvax II and down-loaded into a slave $\mathrm{KXT}-11$ processor using a software loader purchased form Digital Equipment Corporation. This permitted the Microvax II to be the master control computer with the KXT-11 processor being the dedicated slave processor for control of the PASC switching signals. The Microvax II was also used to collect and store the experimental data from the PASC Feasibility Demonstartion system. For a three phase power system it would require ore KXT-11 slave processor per phase if a eight-pulse height system is to be constructed. The required control configuration for single phase is shown in Figure 5.

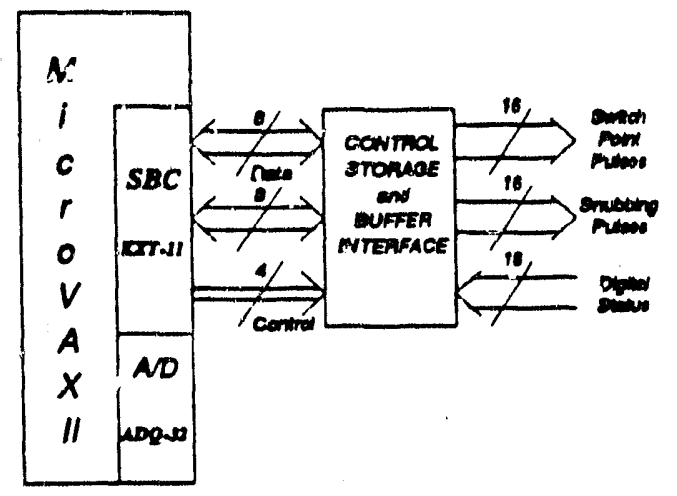

Figure 5. DEC Microvax IIMaster Controlier with KXT-11 SBC Slave Switching Control Sequencer

Also shown in Figures 3 and 5, is the KXT-11 Interface and Gate firing circuit. Interfacing with the 20-line parallel I/O port signals generated by a Z-CIO (28536 Counter/timer and Parallel I/O), chip on the KXT-11 board and the PASC switching circuitry is provided using standatd TIL IS series logic units. The 8-bit A and B port signals are stored in two 16-bit registers made up of tri-state octal D-type transparent latch units (74LS373), under controls generated by the 4-bit C-port signals. The 32 outputs of these registers (SA0-SA 15,SBOSb15), contain the control information for each Gate-Tum-Off (GTO), thyristors in the PASC switching systern.

Each GTO switch point is controlled by a Gate-Firing-Circuit (GFC). The 32 outputs from the 16-bit registers drive the optical isolator units of the GTO GFC's. These optical units provide the isolation required between the control signals of the various GTO switch points. The isolator output drives a buffering stage which in tum drives the complementary symmetry MOSFET driver of the GTO gate-cathode circuit. The MOSFET driver provides low-impedance turn-on (.3 amperes), and turn-off ( 1 ampere), sources required for the high speed switching of the low power GTO units. GFC power is supplied by isolated auxiliary plus or minus 9 vdc supplies. A resistor change on the GFC board allows approximately 1 ampere of tum-on driver required for the high power GTO units.

After the need for symmetry became known (both through experimental data and computer simulation), an inteimediate design which produced high symmetry was hand constructed as shown in Figure 6. 


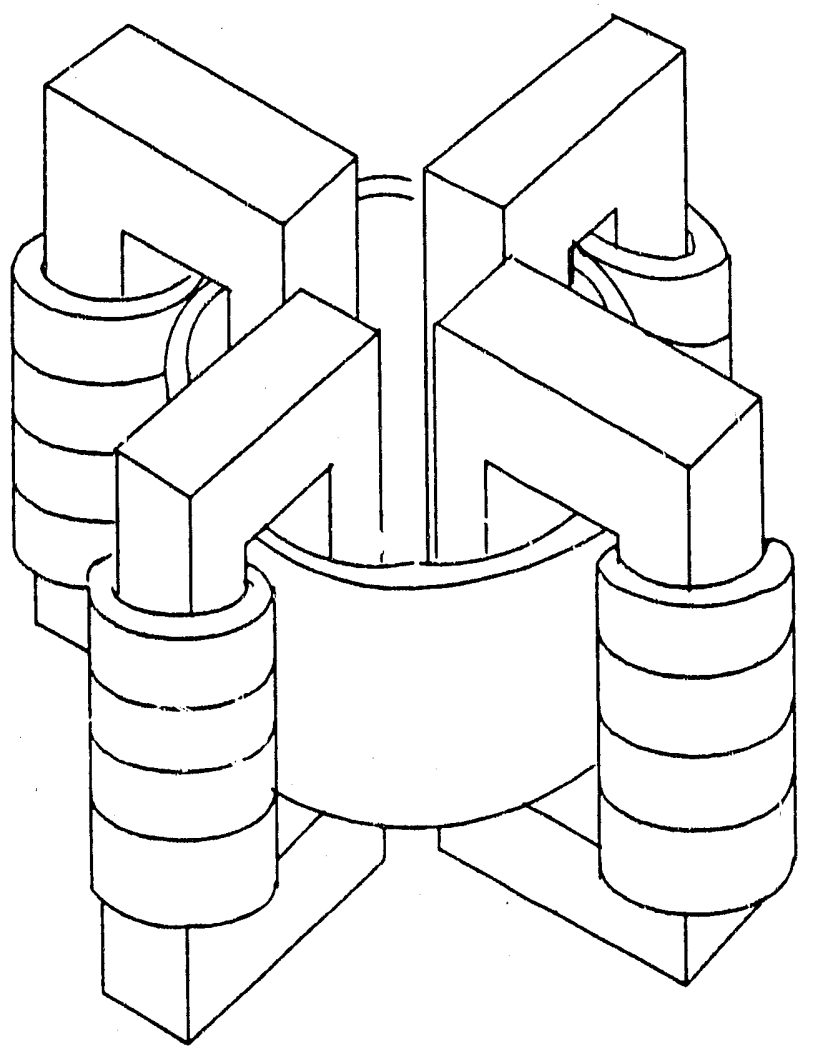

Figure 6. Four Core, 16 Primary, Parallel Secondary PASC Transformer

The original design contained four primary windings per outside transformer web, with a total of 16 primary windings on four webs. This transformer is referred to as a four-core, 16 primary, parallel secondary PASC transformer configuration. All transformers were identically coupled to a single secondary winding occupying the common central web of the transformer. Because of the voltage "leakage" effect (flux is instantaneously conserved, not voltage), when more than a single primary is active on a superpositon transformer, this transformer produced a very poor (non-sinusoidal), output when all of the primary windings are energized. However, when operated with single active prinary per superposition web, the transformer gave excellent results. An additional, optional, central square piece of transformer iron can be added to this design in order to increase the coupling and mutual inductance if desired but further testing of this configuration is necessary in order to validate that this will not result in "voltage leakage" for some input pulse sequences.

Because of a desire to go to a full 16 pulse superposition, and a desire to minimize transformer design and fabrication costs for this research, the multiple web single transformer was set aside in favor of multiple, magnetically isolated but with series, current coupled secondaries. This greatly simplified the computer simulation of the device using either SPICE or our dedicated Fortran programs as well as facilitating the possible use of standard, of -the-shelf distribution transformers for the PASC demonstration design. 
The final configuration chosen for the PASC Feasibility Demonstration design consisted of eight transformers each of which contained two primaries and a single secondary winding. This produced a design in which one primary winding on each transformer was used for generation of positive-going pulses and one primary was used for negative-going pulses, thus achieving the desired result of having only one primary active per transformer so as to avoid the voltage "leakage" effeci mentioned previously. To achieve superposition of the pulse outputs, all of the secondaries of the eight transformers were connected in series as shown in Figure 7.

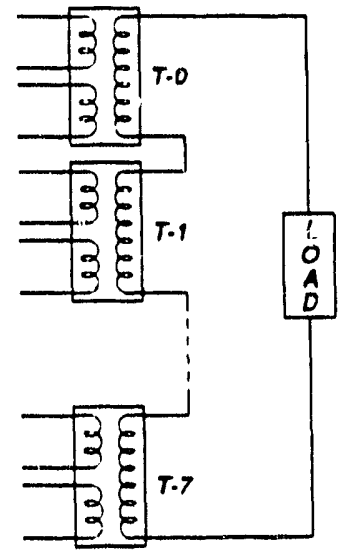

Figure 7. Two-Primary Winding CI Transform.rs With Series Connected Secondaries.

To achieve no voltage leakage occurance for those time intervals when only certain of the transformers are active, all primaries of non-active transformers wisre kept shorted. This guaranteed that the voltage drop across all of the non-active transformer secondaries would be minimal. To test the thesis that voltage leakage could be avoided by shorting of the primaries of all non-active transformers a set of generic transformers which were left over from another research project were used. These transformers produced very satisfactory results but were not useful for the Feasibility Demonstration tests irecause they lacked two primary and two secondary windings so that both step-up and step-down configurations could be tested. They also had a non-ideal turns ratios and values for self inductances. Results for step-down testing of these transformers were so satisfactory that it was der,ided to do the Feasibility Demonstration testing using small standard power distribution transformers similar to the generic transformers but with two primary and two secondary windings and greater primary and secondary inductances. General Electric transformers satisfying these requirements were then ordered and refitted into the PASC demonstration system.

3.2 Preliminary computer simulation results from applying the PASC technology to the diagonal generator connection.

Although not called for in the work statements, the application of PASC technology to diagonally connected MHD generators is of considerable interest for commercial applications if very low harmonic content power frequency waveforms can be easily generated on diagonal configuration MHD generators. One implementation of the PASC concept to MHD diagonally connected generators can be achieved by means of parallel excitation of a group of primary transformer windings whose secondaries are then connected in series as shown in Figure 8. This configuration is a straighitasnward extension of the PASC technology 
already demonstrated for Faraday type connection: the primaries of all of the transformers are connected in parallel across the two-terminal output of the MHD generator rather than individual electrode pairs.

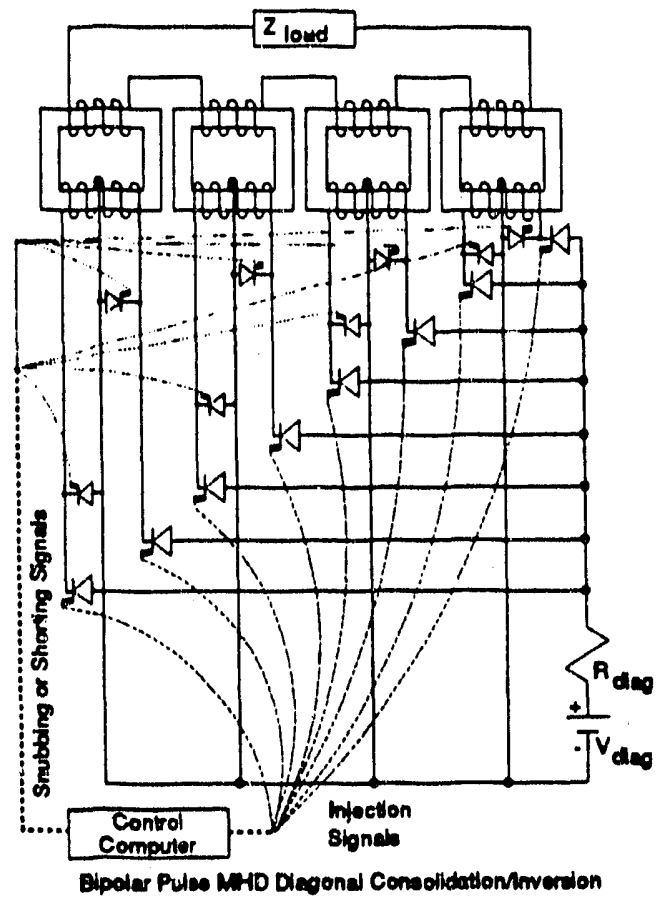

Figure 8. PASC Four-pulse CI System For A Diagonally Connected MHD Generator

Shown in Figure 8 is the circuitry for a single phase inverter waveform synthesis consisting of four positive and four negative pulses which are produced by time multiplexed switching of the eight GTO power injection thyristors indicated. A more typical commercial system would consist of eight transformers in order to achieve desired low harmonic content without filtering. A standard three-phase inverter would be constructed by placing three such circuits in parallel across the diagonal voltage source $V_{\text {dieg }}$. The three single-phase inverters would then be synchronized with the same control computer so as to produce the usual phase shifted three-phase output. All of the required computer switching and control interface technology is identical to that already developed for the SDI PASC Feasibility Demonstration Project; only the software control algorithm must be changed for this diagonal connected PASC application. The synthesis mode indicated in Figure 7 is only one method of carrying out the necessary waveform synthesis; other methods using proprietary circuitry are being investigated.

Results are discussed below for simulations involving a Thevenin equivalent circuit to represent the MHD generator, in which the resistor shown in Figure 8 represents the local ratio of diagonal open-circuit voltage to short-circuit diagonal current. Since no measurable values are directly available for this data (they might be extrapolated from data for some existing generators), per unit dimensionless values in the range of 1 to 100 ohms have been assumed, (see Rosa[1] Fig. 4.6, p. 66). The results shown were obtained using the Electromagnetic Transients Program (EMTP), to integrate the circuit configuration given. 
The results indicate that a sinusoidal load voltage and current can be obtained if the internal Thevenin series resistance is small in comparison with the load voltage. Even if the internal impedance is higher, sinusoidal output can be guaranteed by placing a smoothing electrolytic capacitor across the diagonal terminals. Although no closed loop harmonic suppression control is applied, the resulting waveforms have very low harmonic content, particularly for the case in which the load impedance is $100 \mathrm{ohms}$ and the internal generator impedance is $1 \mathrm{ohm}$.

\subsubsection{Modeling the PASC Process Using EMTP}

The EMTP[2] program is a standard in the utility industry which is used to study switching transients on power distribution networks and high-voltage transmission lines. It has detailed models for actual power transformers but does not represent power semiconductors well (especially GTOs); these are modeled in the present simulations as ideal switches which is close to reality as far as the load voltagecurrent results are concerned while of course not providing any detail as to GTO internal behavior. SPICE[3] could also be used to model the PASC process but it has very poor transformer models. EMTP's advantage for this study is that it is possible to model the PASC network at the systems level very rapidly without a lot of programming. Because EMTP is usually applied to very large networks with requirements for integrating very large state vectors, it utilizes trapezoidal integration to cut integration time and is absolutely stable but also is known to be inaccurate in local behavior. Thus, based upon other simulations we know that the results presented herein are correct in general behavior but the instantaneous behavior immediately after switching points is somewhat in error because the trapezoidal integration does not handle optimally the opening of switches on inductors. We can compensate for this by placing high values of resistance across the switching contacts (that is by introducing dissipation), but the values are then somewhat attenuated with respect to the correct solution. Thus in the results which follow the sharp spikes or local oscillations in the waveforms are incorrect; both Runge-Kutta and Adams-Bashforth integrations of a very few simulations have verified this), but the gross or average value of the waveforms is quite close to the correct values.

As can be observed in all of the results, the sharp sawnooths in the waveform only occur at the switch opening points on the inductors. Tr is behaviour is major reason that a more robust integration algorithm is planned fi the next release of EMTP.

\subsubsection{Discussion of Results:}

The results show the strong effect of (source impedance)/(load impedance) ratio on the resulting waveform. All results shown are for a transformer step up ratio of $1: 2$ and are the result of superimposing the outputs in series of eight transformers secondaries. No attempt has been made to perform any control on the superposition widths of the pulses making up the waveform other than the standard sinusoidal modulation which has been used for the Faraday PASC configuration of eight similar transformers. Thus considerable additional harmonic suppression capability is possible beyond the present results. The only waveform that is really acceptable is for the case of $R_{\text {source }}=1 \Omega, R_{\text {load }}=100 \Omega$. As previously stated, no attempt has been made so far to model the MHD generator in a more realistic manner. 
Figure 9 show the output current for a Thevenin source resistance of $10 \mathrm{ohms}$, and a load resistance of one ohm. The primary source impedance relects into the secondary by the step-up turns ratio squared, or $\mathbf{4 0}$ ohms. Thus for the series secondary connection, as the number of active transformer secondaries increases with time during the sinusoidal synthesis process, they couple to the secondary side of the transformers greater and greater amounts or reflected primary source impedance until this reflected impedance dominates the the secondary load impedance. This coupled or time dependent secondary impedance depends only on the source impedance and the turns ratio of the transformers. During the rise from one active transformer to eight transformers, the reflected secondary transformer impedance increases from 40 to $320 \mathrm{ohms}$. Hence, for the source impedance of ten ohms, all loads except for very large load impedances, (and hence very low loading) the response shown in Figure 9 is to be expected and will be unsatisfactory for commercial application.

Figures 10, 11, and 12 are for a source impedance of $1 \mathrm{ohm}$ which reflects into the secondary as 4 ohms for a single transformer to $32 \mathrm{ohms}$ as all eight transformers become active. Thus for the case shown in Figure 10, with a load impedance of $100 \mathrm{ohms}$, the load impedance at a minimum is still more than three times the maximum reflected source impedance and the resulting load current is nearly sinusoidal in form. In the cases indicated in Figures 11 and 12, the load impedance becomes less than $1 / 3$ and less than $1 / 30$ respectively of the maximum reflected source impedance with again, highly non-sinusoidal output.

In summary, what does this say about power from a diagonally connected MHD generator? The source impedance of a MHD generator is quite low, varying typically from .005 ohms to .2 ohms for liquid metal to gaseous coal fired MHD generators. Hence if the diagonal voltage is not too low, a relatively moderate voltage step up will be required to meet utility conditions and thus there should not be any trouble guaranteeing very low harmonic content for this $\mathrm{C} / \mathrm{I}$ technology. As a matter of perspective, the internal impedance of most large commercial ac generators is of the order of one ohm so that they should be at least as poor as MHD in this regard. However, they now typically generate directly up to 26,000 terminal volts which is somewhat beyond most present MHD designs.

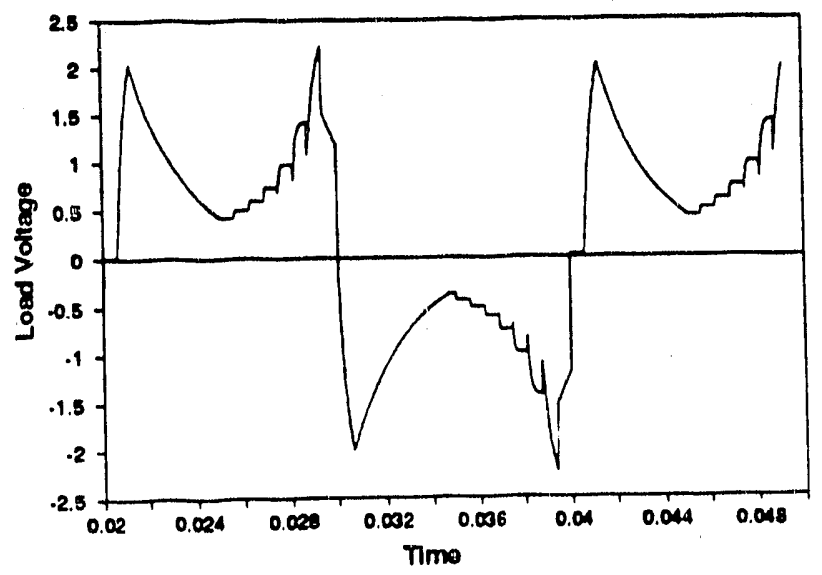

Figure 9 
Dlegonal Conflg. Reources=1, Aload =100

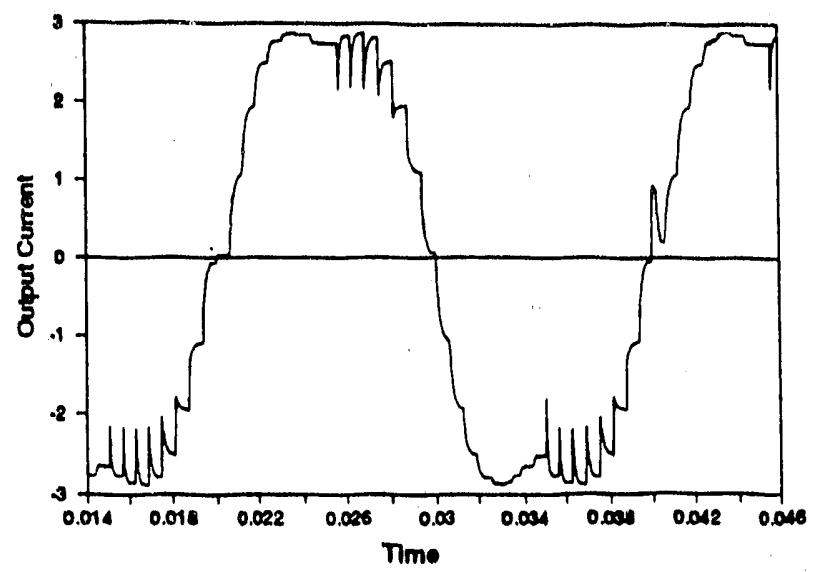

Figure 10

Diagonal Conflg. Rsource=1, Rload=10

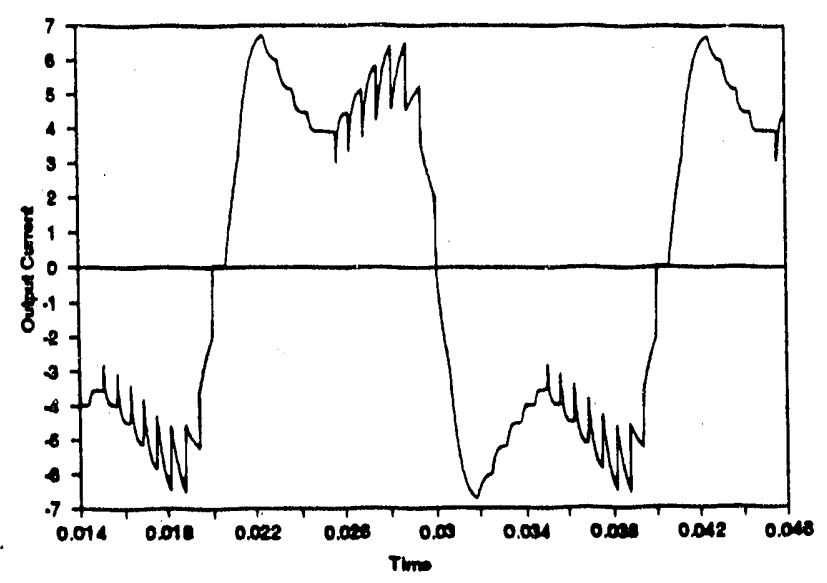

Figure 11

Dlagonal Config. Reources=1, Rloadz1

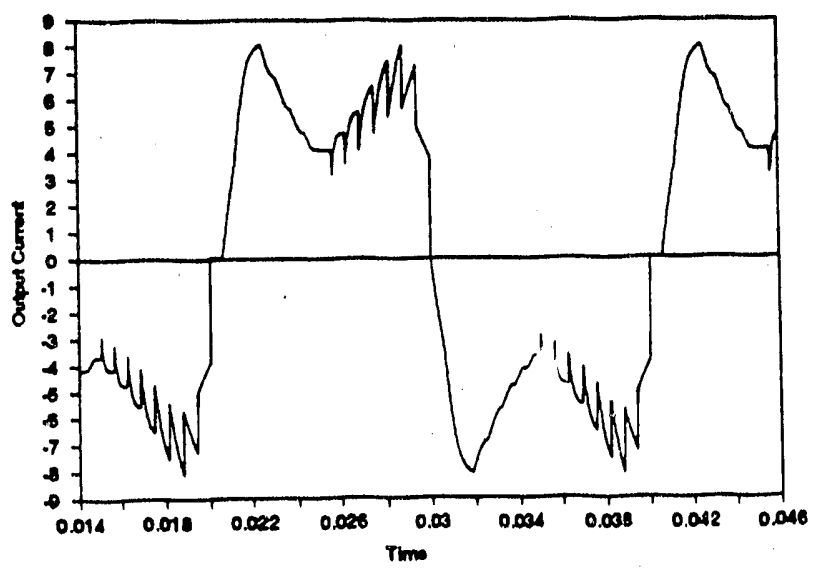

Figure 12 


\subsection{Feasibility Demonstration Test Results Using Low Power Semiconductors}

The Feasibility Demonstration Test of the PASC technology was conducted on Wednesday, August 16, 1989 in the Electrical Engineering Laboratories at Montana State University with representatives from the PETC DOE/MHD office, W.J. Schaffer Associates, support contractor for DOD MHD/SDIO, Mountain States Energy, and DOE/INEL. Although the results were reported previously, the Feasibility Demonstration Test Agenda and Results were performed with low-power GTO semiconductor switches. The agenda and results of the low power tests are summaraized here for completeness and for comparison with the intermediate-power test results given in section 3.4. Detail result figures for the low power semiconductor tests were given in previous reports.

The scheduled agend included:

a. A presentation on the theory and design of the PASC hardware sysiem, the computer control and programming system, the computer switching - GTO interface, The source d.c. power supply design, the GTO switching power supply design, preliminary simulation results of several input waveforms, and results obtained previously using generic transformers in parallel and series connections.

b. A laboratory demonstration of the PASC system operating with the new transformers connected in series secondary connection, step-down voltage configuration. Responses using both high and low impedance snubbing, variable programmed output waveform and frequency, and variable output load impedance were demonstrated.

c. Following a break to permit re-connection of the transformers in step-up configuration, the laboratory demonstrations were repeated. Finally, several pulse waveforms not called for in the work statements were demonstrated.

d. Following the demonstration, a session devoted to requirements for possible application to various weapon systems was held. These included generic FEL and NPB type devices.

e. Finally, an exit session was held in which PETC DOE/MHD contract technical management indicated that an estimate of the power/weight ratio for a PASC power conversion system at the $100 \mathrm{MEW}$ level would be useful.

The waveforms demonstrated in detail included algorithms approved as part of the Feasibility Demonstration Test Plan. Programming of the KXT-11 slave GTO control processor was demonstrated by performing several test algorithms called for in the statement of work.

These algorithms included:

b.1 A $60 \mathrm{~Hz}$ symmetric sawtooth waveform constructed from the superposition of eight positive pulses and eight negative pulses. Since the required delay between pulses to produce this waveform is uniform, it will sometimes be referred to subsequently as a constant delay triangular waveform.

b.2 A $60 \mathrm{~Hz}$ sinusoidal approximation waveform constructed from eight positive and eight negative pulses which are switched at time delays to give a best fit to the sinusoidal waveform if the system is behaving linearly. 
b.3 A zero delay symmetric waveform constructed from eight positive and eight negative pulses which are gated on sequentially at the n.aximum rate that the KXT-11 can perform. Since the delays are all the same number of KXT-11 machine cycles, the resulting waveform turns out to be a sawtooth wavform at approximately $600 \mathrm{~Hz}$.

b.4 A very slow cycling of the GTO switching cle sequence, not intended for powered operation, but for checking the proper sequencing of main and snubbing GTO switching signals by means of a iight emitting diode array.

b.5 In addition to the above waveforms, both a bipolarity rectangular pulse waveform and a bipolurity ramp waveform were generated.

Loads for which successful waveform synthesis operation was demonstrated included: open circuit, $100 \mathrm{ohms}$, and $10 \mathrm{ohms}$. Linearity of response was demonstrated at all load conditions in step-down connection configuration while step-up configuration demonstrated linear response to open circuit and $100 \mathrm{ohm}$ loading with non-linear compression at the $10 \mathrm{ohm}$ loading. The compression in at the $10 \mathrm{ohm}$ loading is caused by the very low impedance reflected into the d.c. Source side of the PASC transformer with a resulting large voltage drop occurring across the Thevenin source impedance rather than the PASC transformers.

\subsubsection{Summary of PASC Feasibility Demonstration Low-power Test Results}

3.3.1.1 The master/slave computer control switching system perfonns as designed and programmed. The KXT-11 slave switching processor can successfully generate sinusoidal algorithm commutation pulses with variable delay requirements up to approximately $300 \mathrm{~Hz}$ for 16 primary GrO switches and 16 snubbing GTO switches. With no time delays (linear triangular pulses), the maximum frequency that can be switched is approximately $600 \mathrm{~Hz}$. Faster, software compatible processors are available which can boost this rate to approximately $1.8 \mathrm{KHz}$ for general waveform synthesis requirements. For d.c.-to-d.c. conversion synthesis requirements for two terminal diagonally connected MHD generators, which are much less general in their real-time speed requirements than Faraday connected generators, it is possible to go to at least $50 \mathrm{KHz}$ rates with the proper control microprocessor. However, GTO duty cycle limitations along with switching and transformer core losses will generally limit such rates to below 10 KHz.

3.3.1.2 Virtually any waveform requirement within reason can be successfully generated using the PASC technology. For highly non-linear loads or loads requiring sophisticated startup and shutdown cycles, closed-loop feedback control of the switching algorithm will in general be required. Output circuitry inverter design must be used if continuous mono-polarity d.c. pulse waveforms are to be generated, because transformers are fundamentally bipolar devices.

3.3.1.3 The efficiency requirements for snubbing or shorting in order to push the energy out the transformer(s) into the load become more severe as the step-up tums ratio becomes high. To achieve maximum efficiency, it may be necessary to use capacitive snubbing or shorting, particularly if the connected load is highly inductive in nature. Such a requirement is much less severe for d.c.-to-d.c. switched mode voltage conversion operation. 
3.4 Feasibility Demonstration Test results obtained using intermediate-level power semiconductors.

Following the original Feasibility Demonstration tests which were conducted in August of 1989, high-power, symmetric GTOs rated at plus-or-minus 1200 volts and 80 amperes were ordered to refit the 16 power injection lines, and 16 high-power natural-commutation thyristors to be used as snubbers. Delivery on these units was delayed twice by the vendor until November 1989, with final refitting being finished in December. It was thought that the 16 asymmetric, low-power snubbing GTOs could be replaced with high-power naturalcommutation thyristor units, since the snubbers should be self-extinguishing in steady-state, periodic operation. Testing revealed however, that unless the transient was very well behaved (less than ninety milliamps of current), these snubbers would lock up with resultant incorrect and damaging performance. Hence the 16 asymmetric, low-power snubbing GTOs were put back into operation because it was thought that in steady-state operation they would be of sufficient size to permit operation of the Demonstration system at full power (input injection resistance of $2.5 \mathrm{ohms}$ minimum), and because of the long delay in getting more units (we in fact could not get any vendor to commit to a guaranteed delivery schedule for $m$. : of the high-power units). This long delivery schedule is particularly true of symmetric $v$ ', ttage capability GTO units; asymmetric units in which the voltage standoff in the reverse bias mode is very low are more readily available because of there usage in motor control systems where the incorporation of a series blocking cliode with the attendant higher circuit loss is considered to be less important than the reduction in cost of the GTO.

Figures 13 through 20 show data obtained during intermediate level power runs during February and March 1990, with the power supplies set at 120 vde source voltages. Source resistance values of 10 and $2.5 \mathrm{ohms}$ for loads of 10 and 100 ohms were employed to obtain these output waveforms. Both step-up and step-down transformer connections were tested and the results are indicated.

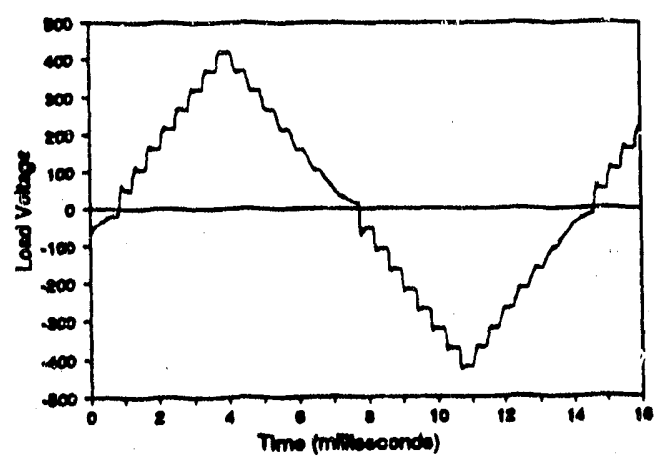

Figure 13. Load voltage for $2.5 \mathrm{ohm}$ source impedance and $100 \mathrm{ohm}$ load impedance for step-down transformer connection. 


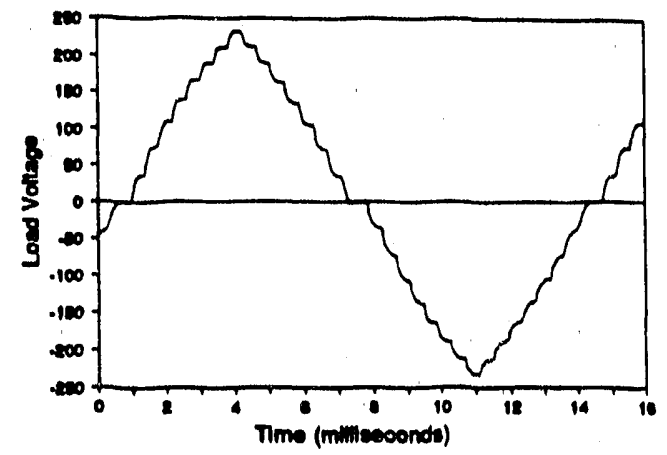

Figure 14. Load voltage for $2.5 \mathrm{chm}$ source impedance and $10 \mathrm{ot} \mathrm{m}$ load impedance for step-down transformer connection.

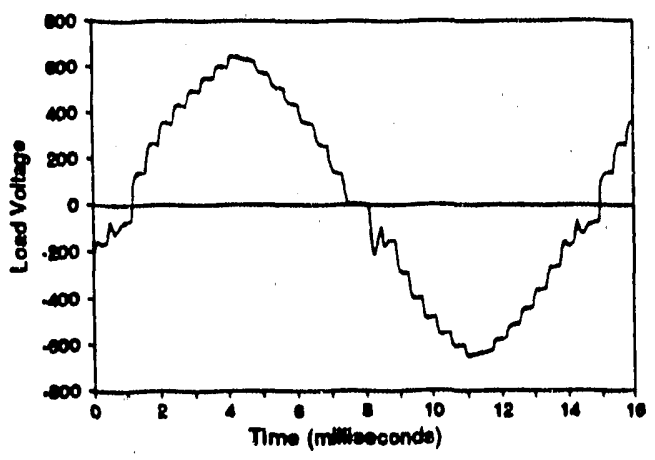

Figure 15. Load voltage for $2.5 \mathrm{ohm}$ source impedance and $100 \mathrm{ohm}$ load impedance for step-up transformer connection.

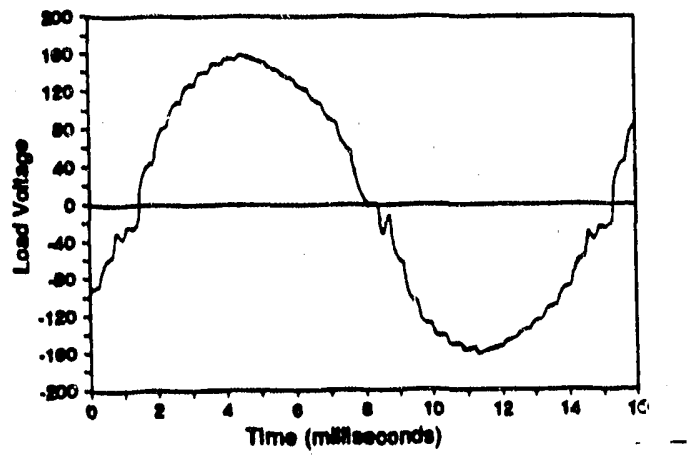

Figure 16. Load voltage for $2.5 \mathrm{ohm}$ source impedance and $10 \mathrm{ohm}$ load impedance for step-up transformer connection. 


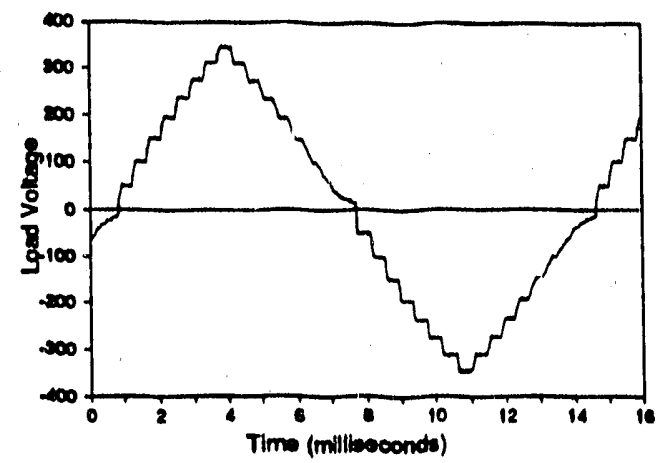

Figure 17. Load voltage for $10 \mathrm{ohm}$ source impedance and $100 \mathrm{ohm}$ load impedance for step-down transformer connection.

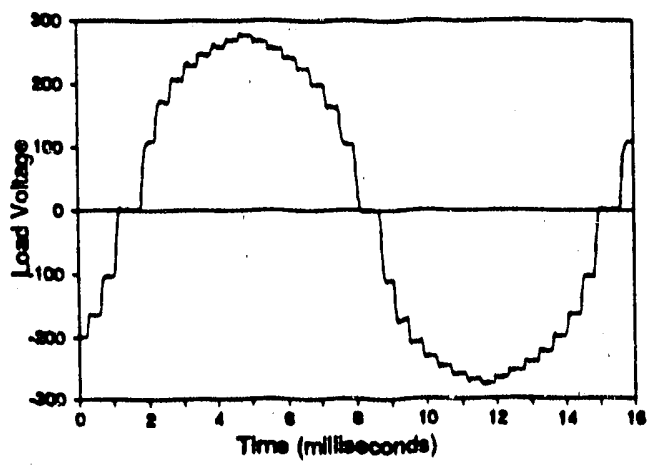

Figure 18. Load voltage for $10 \mathrm{ohm}$ source impedance and $100 \mathrm{ohm}$ load impedance for step-up transformer connection.

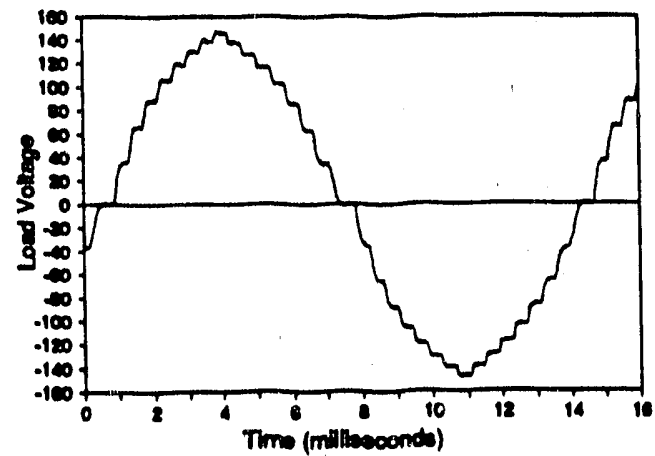

Figure 19. Load voltag: for $10 \mathrm{ohm}$ source impedance and $10 \mathrm{ohm}$ load impedance for step-down transformer connection. 


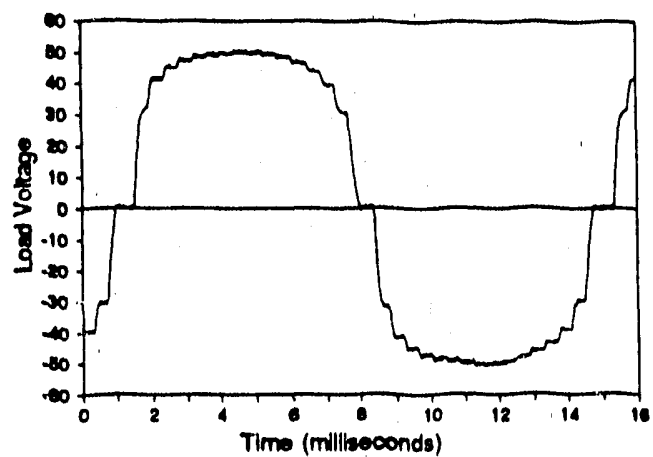

Figure 20. Load voltage for $10 \mathrm{ohm}$ source impedance and $10 \mathrm{ohm}$ load impedance for step-up transformer connection.

The output voltage data scales by a factor of two the data collected using the $60 \mathrm{vdc}$ sources used in the low-nower Feasibility Demonstration testing results obtained during August 1989, discussed above in section 3.3. The general characteristics of the results obtained during February 1990 are similar to those obtained previously with the following additional comments:

a. The low rower GTO snubbing units are being used at or beyond their maximum ratings, especially with respect to maximum current; this produces the anomalies shown in Figures 15 and 16 where two snubbers GTOs are not working properly. It can be seen that suubber number zero (very positive peak of the waveform), and number eight (rigl.c after the waveform goes negative), are malfunctioning. It was ascertained that snubber zero had completely failed. In the case of snubber eight it was determined that it is partially failing. After replacement the tests were repeated with the same snubbers again failing after a very short interval. It must be emphasized that these tests were taken beyond the safe operating ratings of the snubber GTOs. The source resistors also became too hot to touch within five seconds of the start of these tests so that protracted experimentation evaluation of these anomalies is well as reducing the source impedances to zero were not consdidered to be prudent.

b. In general the results for step-down transformer connection for the intermediate level power tests are better than for step-up connection. This agrees closely with that already obtair ed in the low-power demonstration tests. The output waveforms are also better for large load impedances than for low load impedances. Again this agrees closely with previously results and is logically explainable using the exact same argument as given in section 3.2 for the diagonal generator simulation results.

c. Because of the extreme sensitivity of the tests to failure of the low power GTO snubbers, intermidiate-level power data of non-sinusoidal waveforms was not attempted. 
3.5 Description of some preliminary harmonic suppresion control waveform algorithms and a new data acquisition system and control system.

\subsubsection{Introduction}

A question of significant interest in the application of the PASC principle to commercial power production is whether a digitally generated sirusoidal waveform approximation may be controlled in such a fashion as to minimize the higher harmonic content of the synthesized output. In this section we present an application of the Internal Model Principle of feedback control whereby a closed-loop system incorporates an "internal model" of a known reference input for robust tracking of the reference and for improved disturbance rejection. Applying this strategy to a Pulse Amplitude Synthesis and Control (PASC) system requires tracking a sinusoidal reference with minimal error while simultaneously observing other performance measures and design constraints. An inherent feature of a PASC system is the discrete switching of sources to form the pulsed wave. To this end, it is natural to consider a discrete-time control algorithm in which parameters can be easily changed and source switch points are directly available in binary at the output of the controller. The development of the discrete-time Internal Model principle follows closely the continuous-time case given by Francis and Wonhar: in [4].

In what follows, the Internal Model principle is first introduced. A continuous-time system is then built and tested to demonstrate the validity of the model. The closed-10op system is then discretized and a nonlinearity is added at the input to the discrete plant to represent the switching action of the PASC system. Design considerations are ther discussed and some generalizations are made about the control action to be applied. The system is then simulated and analyzed for tracking and harmonic content. Finally, conclusions are drawn and suggestions made for directions in further work.

\subsubsection{The Internal Model Principle}

The Internal Model principle of control theory states that a "structurally stable" design always incorporates 9 model of the reference to be input and a model of the disturbance to be rejected in the feedback path. Francis and Wonham [4] give a general proof of this principle and Franklin and Powell [5] apply the principle to a number of different signals including a sinusoidal reference. Perhaps the most convincing examples of the principle can be found in elementary control theory. It is well known that a Type I system can track a step imput with zero steady state error. Type I systems are easily recognizable in that they contain a single pure integrator. In the sense of the Internal Model principle, however, a pure integrator is a model of a step input. Similarly, a Type II system contains a model of a ramp input or a double integrator. Francis and Wonham prove that an extension can be made to the general case in which the input is an arbitrary function of time. One only needs to find a model of the input in order to be able to track it. It would seem natural that the extension to the sinusoidal reference input case could be modeled by an oscillator at the frequency of the sinusoid. In fact, Franklin and Powell show that this is true. The internal model of a sinusoidal reference is shown in Figure 21. 


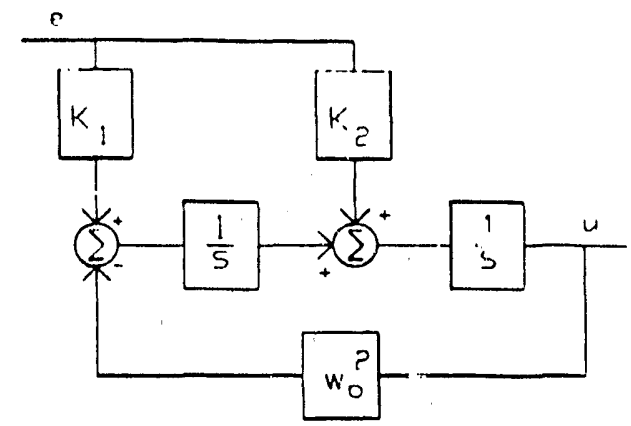

Figure 21. Internal Model of Sinusoidal Reference

Practical experience and testing have shown that a reasonable model for the PASC system under certain operating conditions is simply a single, two-winding transformer with an n-step "staircase" source. If that staircase source is modulated so as to pr uduce an uncontrolled output such as shown in Figure 25, from a simulation standpoint the model is equivalent to using a set of four separate transformer each having two primary windings and a single secondary with all of the secondaries connected in series. This second realization of a four pulse PASC system is of course the way the system must actually be realized in practice because of the voltage leakage principle. The range of valid applicability for this model is low frequency operation in the linear region of the transformer B-H curve and uniform, time-invariant dc source voltages. This is the simplified model chosen to represent the plant for the development of the control strategy. Values for inductance and resistance used in the model are actual test results from the transformers in use in the PASC sy'stem. The transformer model is shown in Figure 22.

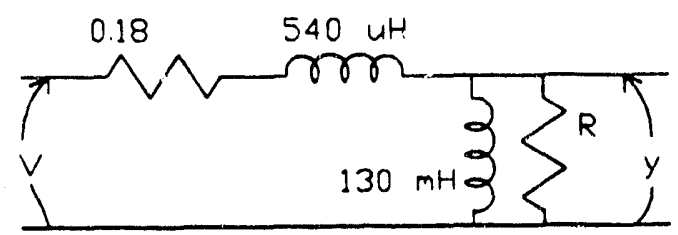

Figure 22. Transformer model for simulation

For further simplification, the transformer tums ratio is taken to be $1: 1$. The transfer function, $G(s)$, representing the transformer can be easily found in the s-plane to be,

$$
G(s)=\frac{1851.85 R s}{s^{2}+(1859.54 R+333.33) s+2564.1 R}
$$

The load resistance, $R$, is left as a parameter which can be varied to perturb the system when the controller is implemented. 


\subsubsection{The Continuous-Time Sygtem}

Figure 23 shows the continuous-time closed-loop system. The switching nonlinearity is represented by the "staircase" in the ncnlinear block. The basic function of the nonlinear block is to quantize the control input. This nonlinearity flects the requirement that only an integer number of dc sources be active at any one time.

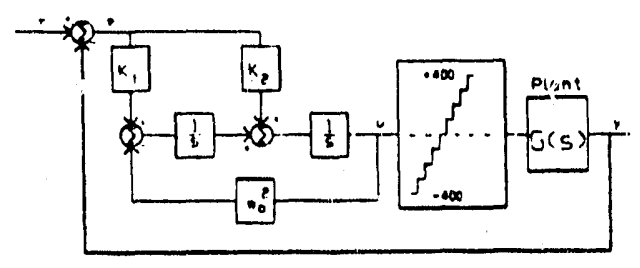

Figure 23. Closed-Loop System

\subsubsection{Discretization of the System}

As was mentioned earlier, it is desirable to use a digital controller in this application. This section presents a discretization of the closed-loop continuous system shown in Figure 23.

\subsubsection{The Internal Model}

The transfer function of the internal model as shown in Figure 21 is

$$
C(s)=\frac{K_{1}+s K_{2}}{s^{2}+\omega_{0}^{2}}
$$

This equation can be split into two parts and easily converted to the $\mathrm{Z}$-domain by table look-up. The discrete transfer function of the controller is then

$$
C(z)=\frac{K_{2}+\left(\frac{K_{1}}{\sigma_{0}} \operatorname{SIN}\left(\omega_{0} T\right)-K_{2} \operatorname{Cos}\left(\omega_{0} T\right) z^{-1}\right.}{1-2 \operatorname{Cos}\left(\omega_{0} T\right) z^{-1}+z^{-2}} .
$$

Since the switching nonlinearity can be easily represented digitally for simulation purposes, a zero-order hold was placed at the input to the plant after the nonlinearity as shown in Figure 24. Again, obtaining the discrete equivalent of the s-plane transfer function is straightforward and can be accomplished by table look-up methods. The z-plane transfer function of the plant and zero-order hold is

$$
G(z)=\frac{1851.85 R}{p_{1}-p_{2}} \frac{\left(e^{-p_{2} T}-e^{-p_{1} T}\right)\left(z^{-1}-z^{-2}\right)}{1-\left(e^{-p_{1} T}+e^{-p_{2} T}\right) z^{-1}+e^{-\left(p_{1}+p_{2}\right) T} z^{-2}}
$$


where $p_{1}$ and $p_{2}$ are the roots of the denominator polynomial of $G(s)$ and are a function of $R$.

\subsubsection{The Discrete-Time System}

We now have enough information to build the discrete-time system shown in Figure 24.

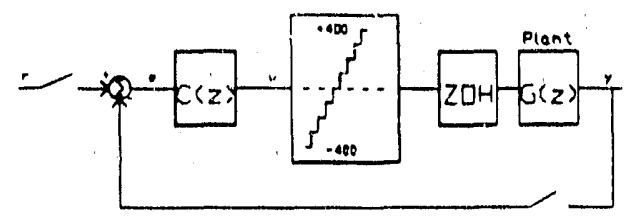

Figure 24. The Discrete-Time System

The system of Figure 24 was tested in various configurations for verification of model parameters using the simulation routines in MATLAB. The results were reasonable representations of the physical system. A case is shown in Figure 25 where the reference input is a $60 \mathrm{~Hz}$ sinusoid with a peak amplitude of 400 volts. The load resistance is $100 \mathrm{ohms}$. Results from the open-loop configuration are shown on the same graph, but are almost indistinguishable from the closed-loop with the controller gains both set at $10^{-2}$. More simulations are shown in the following sections.

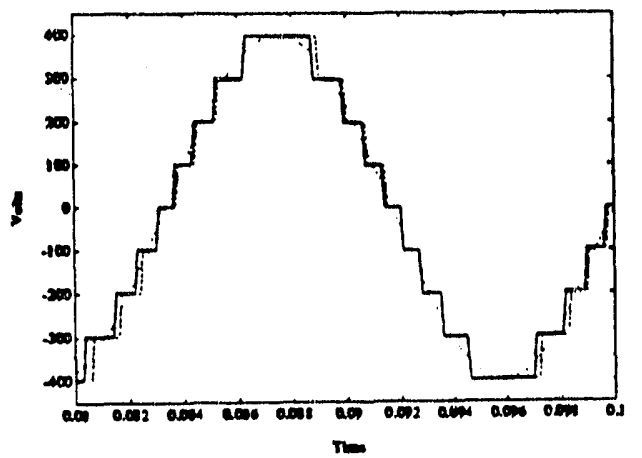

Figure 25. Model and Controller Test 


\subsubsection{Design Considerations}

Numerous sy'stem constraints and performance objectives make it difficult to determine the appropriate gains for the controller. For the purposes of this paper, two performance objectives are considered to be of primary interest. The output should track the reference sinusoid with minimal phase lag and should contain a minimal total harmonic content. The PASC system should have the ability to supply or consume reactive power (VARS) when connected to an extemal network such as a power system. This would be accomplished by setting the reference phase leading or lagging with respect to the network voltage. This is the reason for the importance of tracking the reference with minimal error. The reason for the minimal harmonic content criterion is obvious. In a specific application, there may be many more performance criterion with equal importance.

An important system constraint is the finite switching time required to trigger and commutate a GTO switch. A reasonable estimate of the time required to bring a GTO to full conduction and subsequently commutate it is approximately $20 \mu \mathrm{S}$. For this reason, the sampling rate has been kept at $100 \mu \mathrm{S}$ or greater throughout the simulations. With these in mind, two root loci were constructed in the s-plane to get a feel for the effect of controller gains on the system. The nonlinear block was not included in the open-loop transfer function used to obtain the root locus. The first root locus is shown in Figure 26. In this case, $k_{2}$ is zero and the root locus is constructed on $k_{1}$.

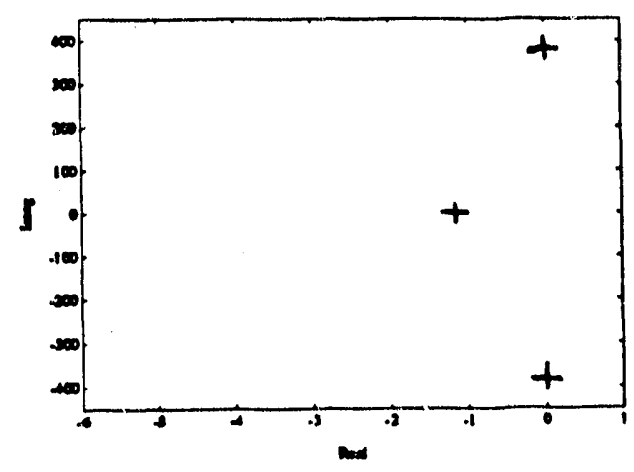

Figure 26. Root Locus on $\mathbf{k}_{1}$

From Figure 26, it is seen that it is extremely hard for $k_{1}$ to move the oscillatory poles off of the $j \omega$ axis. This is understandable by looking at Figure 23 and noticing that with $k_{2}=0, k_{1}$ is feeding a pure oscillator. Figure 27 shows a root locus on $k_{2}$. Here it can be seen that significant damping can be added to the system while keeping the poles at the same oscillatory frequency. Both loci were constructed using 100 gain points between 0.1 and 10 . 


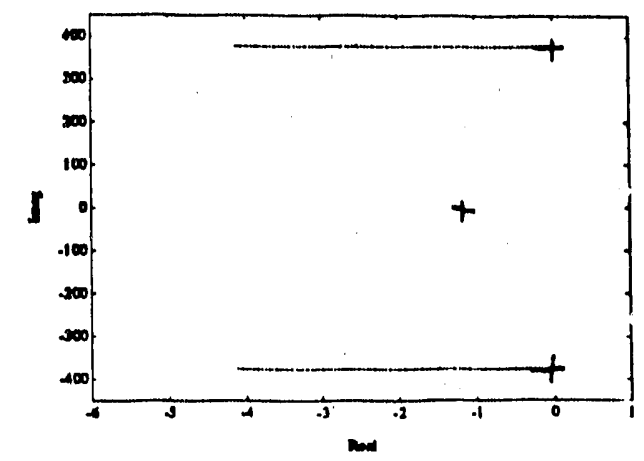

Figure 27. Root Locus on $\mathbf{k}_{\mathbf{2}}$

It will become evident that increasing $\mathrm{k}_{2}$ increases the number of switching actions per ac cycle as the controller trys to track the sinusoid perfectly. This action would fatigue the switches and increase the high frequency content of the output if carried to an extreme. An optimal choice for the gain settings would be difficult to calculate under the physical constraints of the system. Simulations will give a better feel for the choice of gain, but the root lici have shown that $k_{2}$ has a much greater effect on the system closed-loop poles than does $\mathbf{k}_{\mathbf{1}}$.

\subsubsection{Simulations}

Figures 28 and 29 show the effect of increasing the $k_{2}$ gain. In Figure $28, k_{2}=1$ whereas in Figure 29, $k_{2}=10$. In both cases, $R=100$.

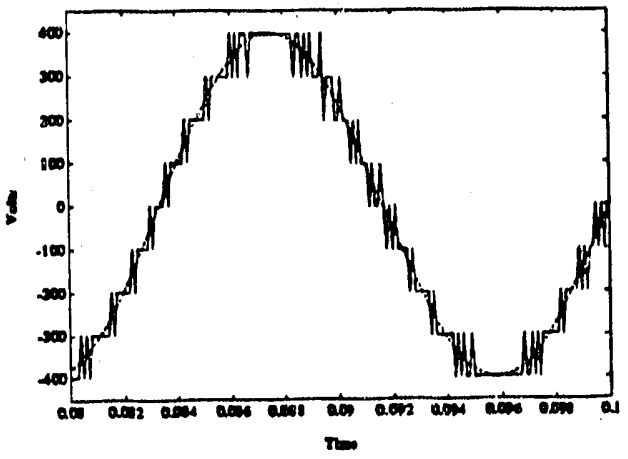

Figure 28. $R=100, k_{1}=1, k_{2}=1$ 


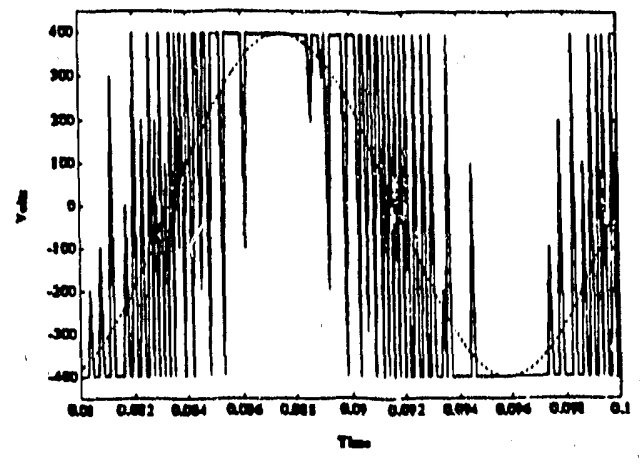

Figure 29. $R=100, k_{1}=1, k_{2}=10$

Figure 30 shows the spectrum of Figure 28 against the spectrum of the uncontrolled plant. The higher frequencies are conspicuous here.

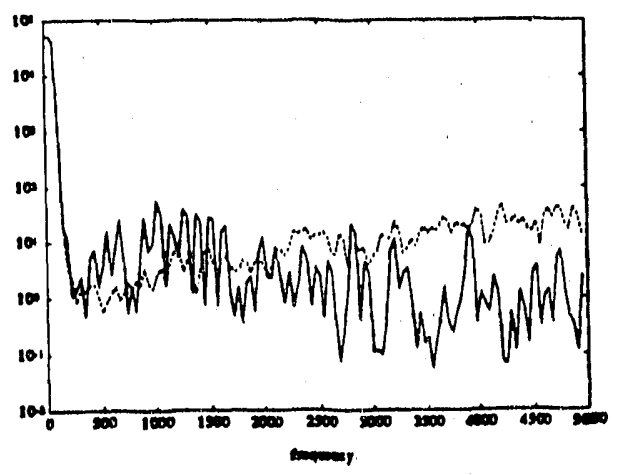

Figure 30. Spectrum of Figure 28

To illustrate heavy loading conditions where the inductance of the transforme: starts to become significant, a simulation was made for $R=1$. Figure 31 shows the outputs of the controlled and open-loop systems with respect to the reference input. Figure 32 shows the spectrum of Figure 30.

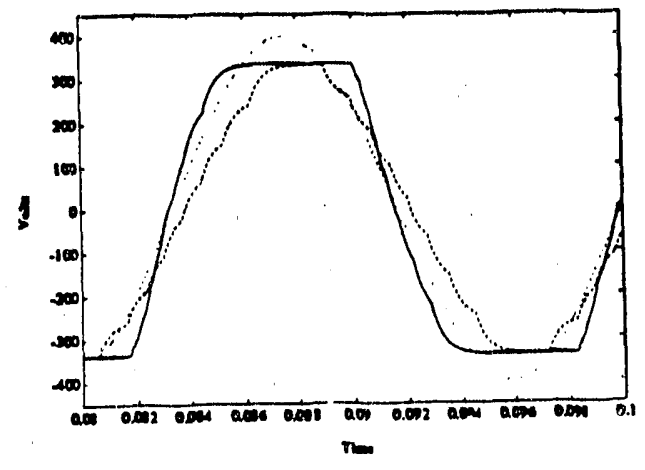

Figure 31. $R=1, k_{1}=1, k_{2}=0.1$ 


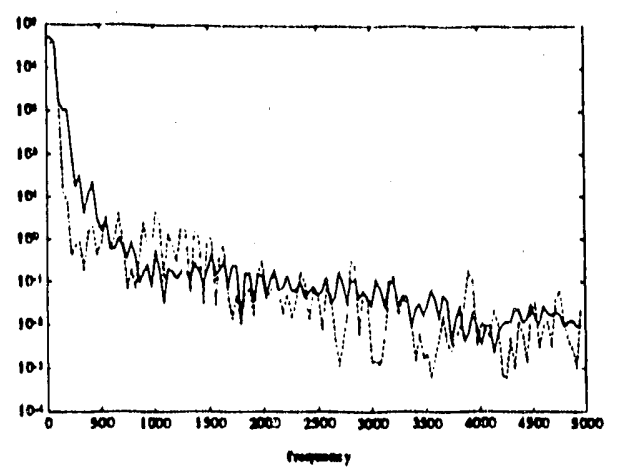

Figure 32. Spectrum of Figure 30

In a third case, the frequency is increased to $600 \mathrm{~Hz}$. Figure 33 shows the output and Figure 34 shows the spectrum.

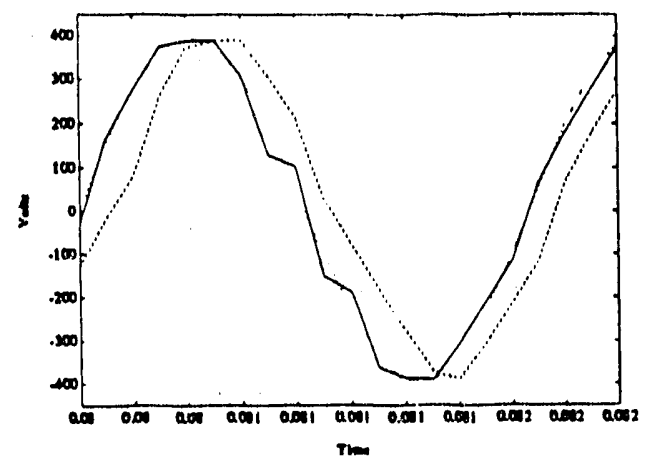

Figure 33. $F=600 \mathrm{~Hz}, \mathrm{R}=10, \mathrm{k}_{1}=1, \mathrm{k}_{2}=1$

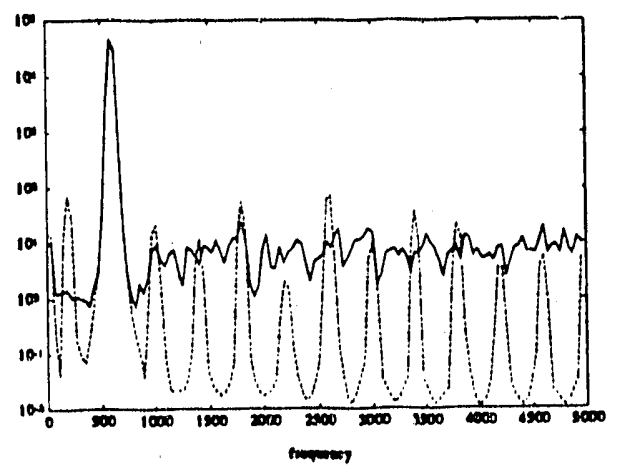

Figure 34. Spectrum of Figure 33

Numerous other simulations were conducted among which were cases where the reference frequency was not equal to the controllers $\omega_{0}$. Extreme attenuation of the output was experienced in these cases where the internal model was no longer a model of the input. 
The Internal Model principle has heen examined and applied to a system with which we would like to track a sinusoidal reference input. The principle is summarized by Francis in saying, "...a structurally stable synthesis must utilize feedback of the regulated variable, and incorporate in the feedback path a suitably reduplicated nodel of the dynamic structure of the disturbance and reference signals." [4]. The development of the model for a sinusoidal reference followed from the work in [4] and from a discussion in [5, p.389]. The continuous-time model was discretized and the discrete system was run through simulations and analyzed for spectral content.

While it is clear that the controlled system tracked the reference input better than the open-loop system in all cases, it is not clear that significant reductions in harmonic content occurred in all cases. At low frequency and low loading conditions, there is a trade-off between low frequency harmonic content and high frequency content in the controlled case. More control provides lower harmonic content below about $600 \mathrm{~Hz}$, but increases then content of the spectrum above $600 \mathrm{~Hz}$. This may suggest an optimal gain for a given loading condition to minimize total harmonics. It may be the case, however, that high frequency harmonics are filtered adequately by the external system or that the cost of filters is much lower at high frequencies and one would simply want to reduce th: amplitude of the lower part of the spectrum at the expense of faster switching.

\subsubsection{The Harris Design Contest Controller}

Early in 1990 the Harris Desigi Contest was announced. This contest provided a small prutotype board to the contestants containing a very high speed control processor chip directly programmable in the FORTH higher level language, a real-time event clock, and with additional space on the board to place additional memory and an analog-to-digital converter. Two of our graduate students entered this contest with the objective of replacing our Feasibility Demonstration Microvax/KXT-11 controller with the Harris board and implementing the internal model controller. From a power/weight ratio measure this promised some improvements. Prior to this the Feasibility Demonstration system was controlled only in the open-loop using a DEC MicroVax II hosting a DEC KXT-11 (PDP-11 based) single board computer. This system was adopted because it allowed software development at maximum speed by permitting implementation of the control sequence algorithm in a higher order language. In this case, the "open-loop" consisted of downloading a pre-programmed switching schedule developed on the Microvax using a cross-compiller, to the KXT-11 and iteratively running the schedule to produce an output waveform. A cumbersome limitation of the KXT-11 processor is that all timing of events was limited to a multiple of the basic instruction cycle, (a real-time event timing clock along with interrupt driven scheduling of switching would aleviate this problem in a production system, but at the expense of greater software design effort). By using the Harris board several improvements in PASC controller design and operation appered possible. New routines and switching schedules could be developed with any ASCI file editor on $\mathrm{PCC}$ and down-loaded directly into the Harris board for direct execution without the necessity for the normal compiller software iteration sequence. To test compatibility of the RTX $2001 \mathrm{~A}$ with the existing hardware, an open-loop switching schedule was written for the RTX. FORTH programming on the RTX $2001 \mathrm{~A}$ proved to be fast and efficient in comparison, particularly with respect to 
the ASIC bus output. Other drawbacks of the DEC controller sequence program chosen, (again dictated because of the development time constraints necessary in meeting the original Feasibility Demonstation tests), included the inability to control phase, amplitude, and/or frequency on-line, the inability to compensate for dynamic loading, and the inability to adjust the sivitching schedule to achieve a secondary goal such as harmonic minimization or component failure. The RTXEB provided for the Harris Design Contest had the potential ability to deliver all of these things as well as the ability to work in parallel with other boards as would be required in a three-phase system.

\subsubsection{The Digital Controller}

With the design goal as stated above, i.e. the synthesis of a 60 hertz sinusoid with minimal harmonic content, the controller design was centered around the internal model principle of control theory. Simply put, the theory states that in order to track a reference exactly or to reject a disturbance completely, a model of the reference/disturbance must be incorporated in the feedback path of the controller [4]. In the application proposed for the Harris Design Contest, the authors wished to track a reference sinusoid. The internal model principle dictates that a sinusoidal oscillator must be incorporated in the feedback loop. The closed-loop system can therefore be represented in continuous time as in Figure 35.

The non-linearity resembling a staircase represents the discrete levels of output voltage available. The number of steps corresponds to the number of transformers connected to form the additive waveform. To implement the controller on the RTX 2001A, the discrete-time equivalent of the oscillator in Figure 35 was developed and written in the form of a digital filter. As a ratio of polynomials in $z^{-1}$, the filter can be written

$$
C(z)=\frac{K_{2}+\left(\frac{K_{1}}{\omega_{0}} \operatorname{SIN}\left(\omega_{0} T\right)-K_{2} \operatorname{Cos}\left(\omega_{0} T\right)\right) z^{-1}}{1-2 \operatorname{Cos}\left(\omega_{0} T\right) z^{-1}+z^{-2}}
$$

where $z^{-1}$ is the discrete delay operator. It can be shown that effective control for this application can be achieved by setting $K_{1}=0$. The software implementation of the filter is carried out using transposed direct form II. Figure 36 shows the closed-loop system in block diagram form as it is operating on the RTX 2001A. 


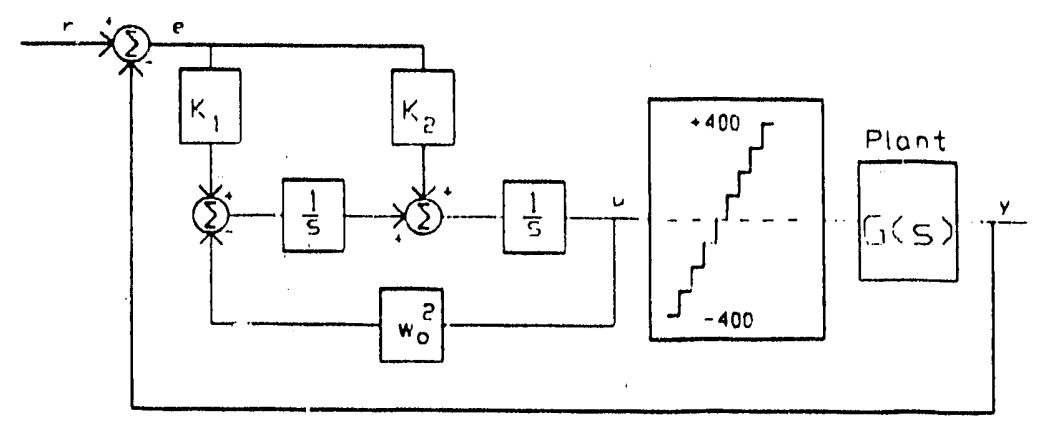

Figure 35. The closed-loop system in continuous time.

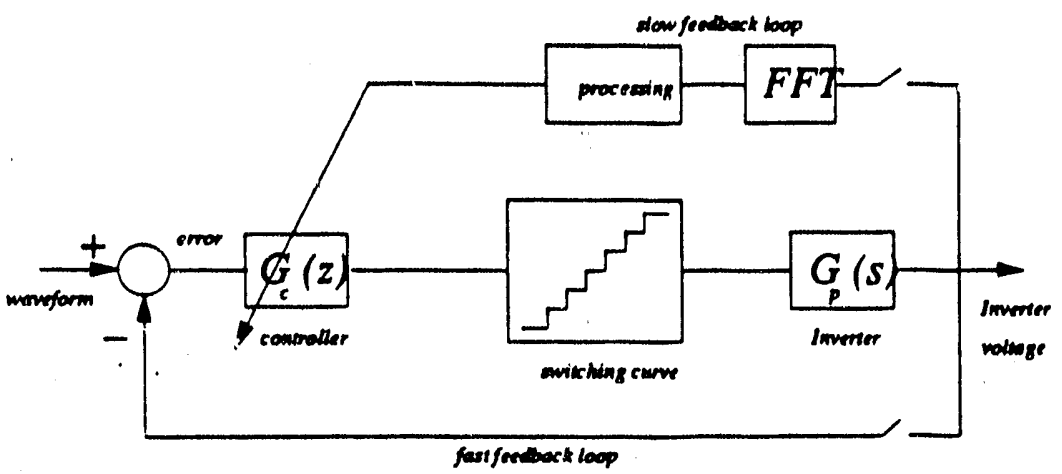

Figure 36. Block diagram of closed-loop system.

\subsubsection{The Continuous Fourier Transform}

The action of the controller was first studied using the MATLA.B software packages before the actual implementation on the system. The results of the simulations confirmed an intuitive guess that increasing the gain of the controller would force the output to track the reference sinusoid more closely thereby reducing the lower harmonics at the expense of more frequent switching actions. The increase in the amount of switching required to track one cycle of the reference leads to an increase in the higher harmonics contained in the output, thus a tradeoff exists between reducing the lower harmonics and th: higher harmonics. The definitions of lower and higher order harmonics is purely subjective. For the commercial utility application, it is al ways desirable to reduce the lower harmonics biecause the higher harmonics tend to attenuate rapidly along a power transmission line or through a power transformer. 
With this in mind, it was suggested that a means be found to monitor the harmonic content of the output and increase the controller gain to a point where the higher order harmonics stay within a reasonable limit and there is no damage to the GTOs by attempting to switch too fast. The continuous Fourier transform outlined in the Harris publication RTXpress [6] proved to be an answer to the problem and the ease with which interrupts are handied on the RTX 2001A made implementation of this feature extremely simple. One CFT is performed on the output at 180 hertz (the third harmonic) and another is performed at 720 hertz (the twelfth harmonic). A ratio of the third over the twelfth is then formed and this ratio is used in a form of adaptive control to adjust the gain of the controller. As the ratio increases, we wish to increase the gain to bring the ratio back down to a relatively constant value which experience has shown to be governed by physical limitations on the switching speed of the GTO's. The CFT's are performed in the background of the normal controller action and are continually being interrupted to perform the control by an on-board timer provided on the RTX 2001A This process is discussed more thoroughly in the section on software implementation.

\subsubsection{Hardware Interface With the Existing System}

The system, as it was constructed for the Microvax realization, utilized 32 TriState drivers, each one driving a single gate firing circuit through an optical isolator. The 16 data lines were multiplexed using a 3 bit address into the 32 GTO gate firing circuits. The simple nultiplexing scheme consisted of one address bit dedicated to " output enable " for supervisory control and the remaining two bits were used as TriState latches to toggle the information into either of two sets of 16 TriStates. This system provides for positive latching of information into the gate firing circuits and provides more speed than will ever be required of the GTOs.

The ASIC bus architecture on the RTX 2001A made the interface to the existing system as easy as could be imagined. The $16 \mathrm{GD}$ data lines are brought directly to the existing TriStates. The output enable bit is held low by means of a toggle switch installed in the prototype area of the RTXEB. The GIO bar and the GR/W bar are inverted and ANDed with the GAO ASIC bus address line. The result of this operation becomes one of the TriState latches while the GA1 line ANDed with the same two " data valid "lines gives the other latch. This leaves one of the 3 external ASIC bus address lines to be used as a Start of Conversion signal to the A/D converter. Figure 37 shows the chip layout on the RTXEB while Figure 38 shows the logic just described.

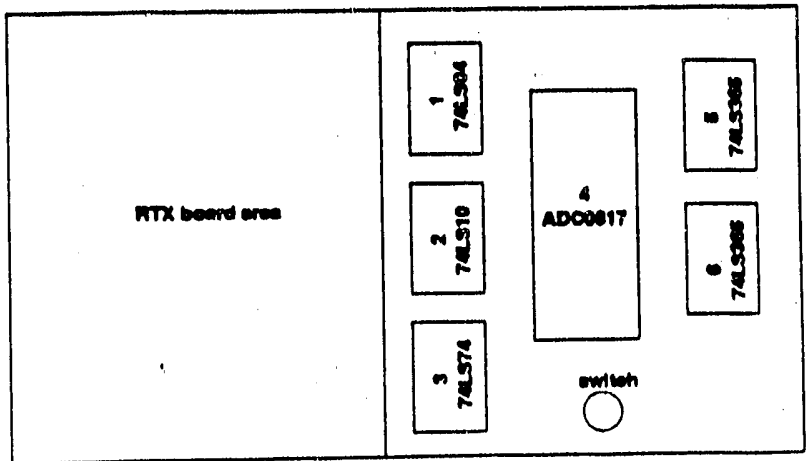

Figure 37. Chip layout on RTXEB. 


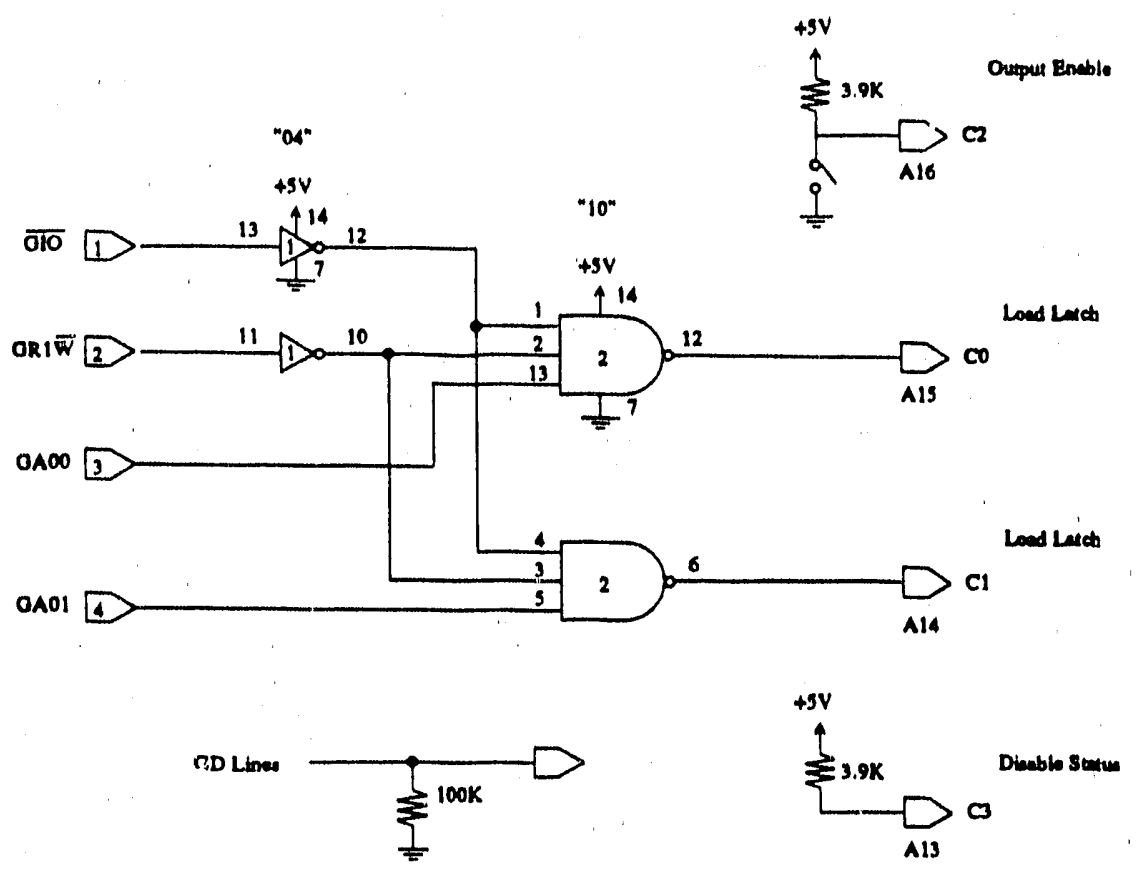

Figure 38. Logic diagram for Mux.

The A/D converter used in the contest application is an 8 bit device running on an independent $1 \mathrm{Mhz}$ clock. Figure 39 shows the clock design as well as the pinout for the $A / D$. Data from the $A / D$ is brought in through the bi-directional GD data lines to the ASIC bus. The A/D is given a Start of Conversion command at each controller time step. The simplest practical method to achieve this is to give the A/D a Start of Conversion signal at each read. This is accomplished with a monostable (one-shot) multivibrator. The one-shot is toggled in the same manner as the TriState latches with the GIO bar, GR/W bar, and the GA2 ASIC, bus external address line. The GIO bar is inverted and ANDed with the GR/W bar and the GA2 lines. This provides the digital controller with feedback containing at most a single time-step delay. The A/D analog data is fed to the board by a standard voltage transducer. The output of the transducer is given a dc offset and is passed through a 2:1 resistive divider located on the RTXEB. 


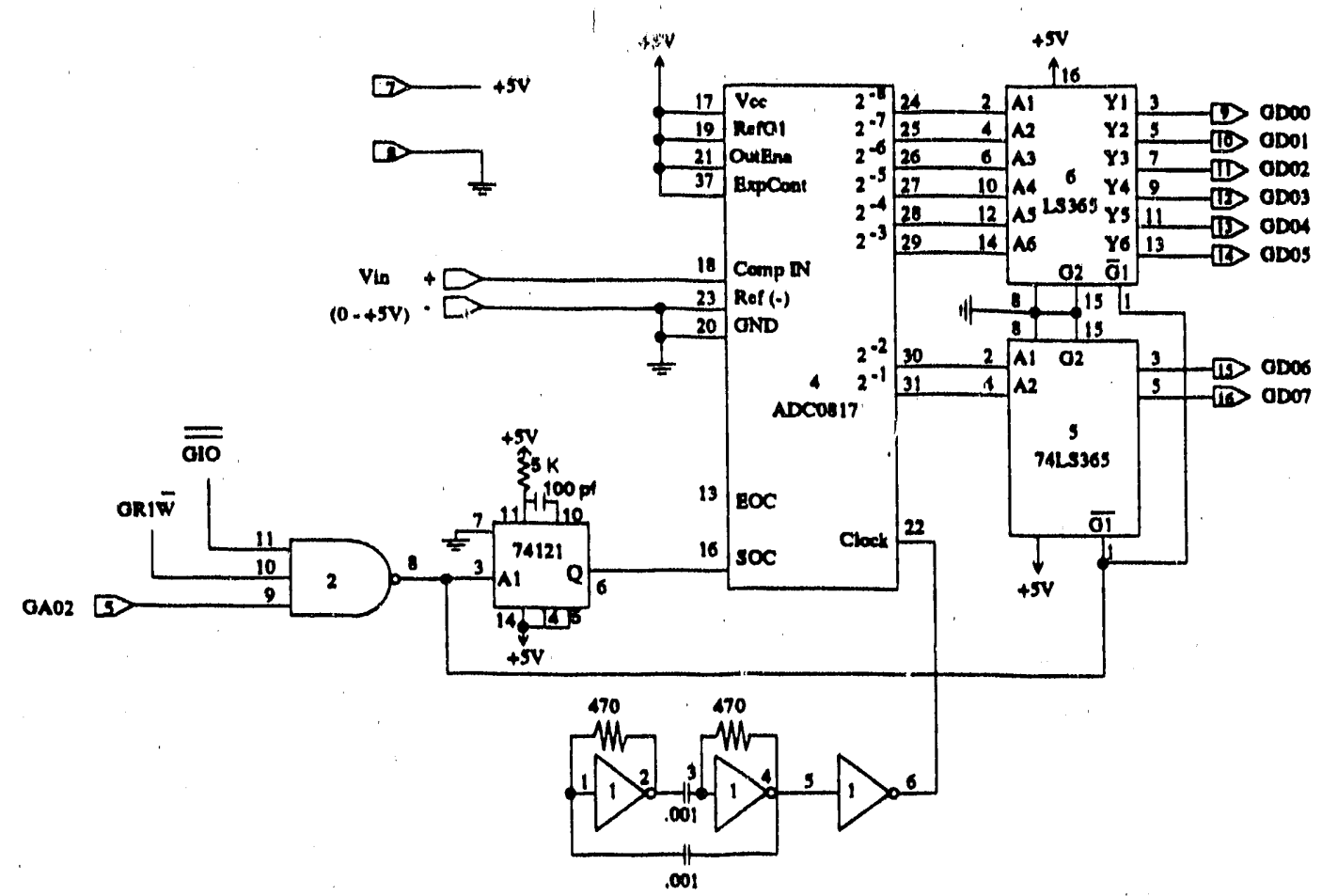

Figure 39. A/D pinout and external clock.

The ASIC bus and GD data lines proved to be extremely easy to work with and minimal time was spend on hardware interfaces. The $3 \mathrm{GA}$ address lines provided give the freedom to multiplex to a greater number of GTOs as would be the case in a system containing more numerous dc sources. In the present configuration, the RTXEB hardware could potentially drive 64 isolated dc sources which is a reasonable number for commercial applications.

\subsubsection{Software Implementation}

The software design strategy for the RTX Design Contest was to implement controller action using vectored interrupts and to run any puripheral processes secondary to the controller. The on-board timers fumished on the RTX 2001A provided the icleal solution to the controller problem. Since the timers decrement once for each clock cycle, the calculations to deternine the timer preset value corresponding to a desired sample rate are simple. The control algorithm is then carried out by loading a timer preset register with a pre-calculated value, unmask the timer, and execute the digital filter at each interrupt. The RTX 2001A timers send an interrupt upon reaching a value of zero and subsequently clear the interrupt and reload to the preset value. To reduce stress on the GTO's, an upper limit of $10 \mathrm{Khz}$ sampling rate was placed on the controller. In an extreme case, this would allow the GTO $100 \mu$ seconds to move into conduction before it could receive a commutation signal to stop conduction. At the $8 \mathrm{Mhz}$ clock rate, $10 \mathrm{Khz}$ sampling corresponds to a preset value of 800 . 


\subsubsection{The Sampling Period and Controller Interrupts}

For reasons of simplicity, the sampling rate was chosen to he $2 \mathrm{Khz}$ or a preset value of 4000 . This rate allows a CFT to be performed at two frequencies between each interrupt. A more optimal strategy which will be easily implemented on the RTX in the near future would be to double the sampling rate to $4 \mathrm{Khz}$ and perform a single CFT between each interrupt. The mode of operation for the Harris Design Contest was chosen so that testing of the prototype could be carried out in a reasonable amount of time and with a significant margin of safety.

The steps involved in handling an interrupt vector corresponding to an execution of the digital filter is shown by the rightmost flowchart in Figure 40. A value for the reference sinusoid is obtained from a data table located in the RTX 2001A data memory. The table is filled with 2008 bit points corresponding to 8 bit values of the first quarter of a sine wave. A pointer points to one of 800 values on a hypothetical sine curve. If the pointer lands in any quadrant of the curve other than the first, a routine called SIN negates a corresponding value in the table or "folds" it or both, depending on what quadrant the pointer is pointing to. In this manner, the number of data points required to fill the table is reduced to one quarter of the number required to represent a full sine period. A table look-up approach was used to determine the values of the reference sinusiod as opposed to direct calculations due to the increased speed of execution of the look-up method.

After the reference point is obtained, a value is retrieved from the $A / D$ converter. This value is scaled and subtracted from the reference to form the error signal shown in Figure 36. At this time, the A/D value is also stored in a table to be used in the CFT. The digital filter is then executed. The output of the digital filter is the theoretical value to be input into a linear system. Since the system being controlled contains the "staircase" non-linearity, the digital filter output must be quantized before being sent to the TriState drivers. The routine called OUTWORD performs the function of quantizing the filter output and sending the quantized value to the GD data lines via the ASIC bus. OUTWORD quantizes the filter output so as to equally load the dc sources under normal operating conditions as best as possible. Control is then retumed to the interrupted nrocedure. The interrupt handler takes about 800 clock cycles to complete. 


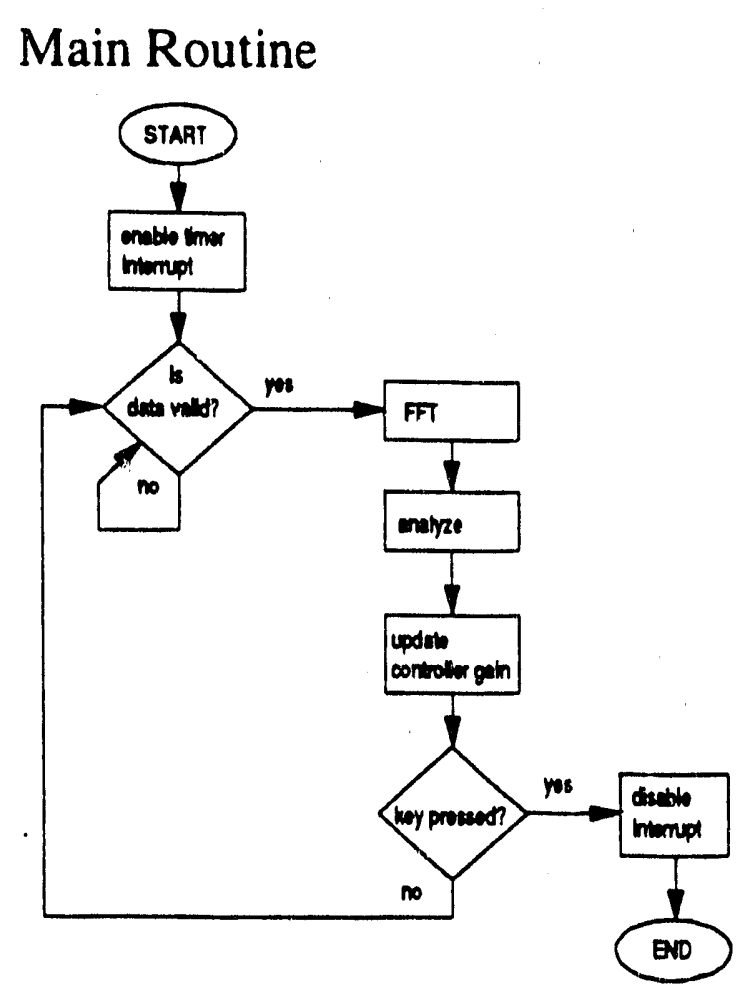

\section{Interrupt Routine}

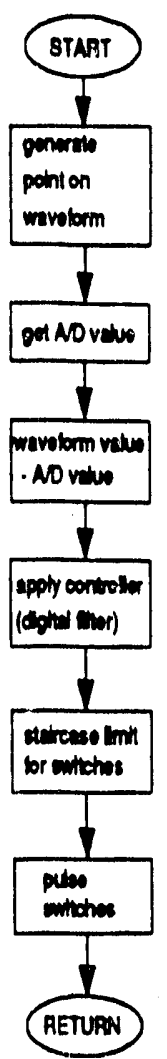

Figure 40. CFT and Interrupt flowcharts.

\subsubsection{The CFT}

The CFT is performed continuously in a BEGIN ... AGAIN structure and is interrupted at each controller time step by an on-board timer. The CFT also contains a keyboard check. Any keystroke caught while in the process of running the inverter will cause a call to a shutdown routine. This routine masks the interrupts, opens all GTOs, and quits the BEGIN ... AGAIN loop to enter the interpret state on the RTX 2001A. Assuming no keystroke was caught end foliowing the procedure outlined in RTXpress [6], the most recent AD value is 
retrieved from the CFT table located in data memory. This is a circular table, so the oldest $A / D$ value is found just above the most recent in memory. This value is also picked up with adjustments for foldover and subtracted from the most recent. This quantity forms the [ $f(k)-f(k-N)]$ quantity which multiplies the exponential. The exponential is broken up into its real and imaginary parts and evaluated using Euler's relation as a cosine and sine. The values of the cosine and sine are picked up from the sine table used in the controller algorithm. The resulting real and imaginary parts of the recursive CFT are squared and added. The square root of this quantity is the magnitude of the CFT. This procedure is performed twice, once at each of two frequencies.

The two frequencies which were determined to be indicators of "lower" and "higher" harmonics for this application are 180 hertz and 720 hertz. A ratio is formed from the magnitudes of the CFT at each of these frequencies and this ratio is used to determine any adjustment needed to the controller gain. Safety features included in the CFT are a flag to indicate that new data is available in the CFT table. This flag prevents the CFT from performing calculations on the same set of data repeatedly. An overflow flag is also included in the squaring operations. If an overflow is detected at this point, the CFT is re-initialized and waits for the CFT table to completely fill up before a new calculation is started. There are many options available to improve the performance of the CFT. Some of these are discussed in the conclusions. As implemented here, the full CFT takes about 2000 clock cycles to complete.

\subsubsection{Memory Allocation}

The philosophy behind the memory allocation on the RTXEB was to first design the controller and CFT to determine how much memory was needed for code space. This being done, most of the rest of the available memory could be used for data. Two data tables are needed in the design. A 200 point sine reference table provides as much accuracy as is being retrieved from the A/D. This leaves enough room in memory for a 1000 point CFT table. Figure 41 show's a map of the RTXEB SRAM available and how it is allocated in the design.

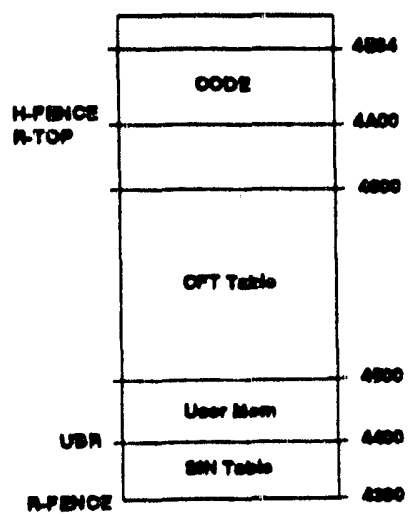

Figure 41. Memory map. 


\subsubsection{Conclusions and Discussion}

The Harris RTX 2001 A proved to he much easier to work with than had been anticipated. As novice FORTH users, the project was approached with a certain degree of apprehension. Features that made the board easy to work with from a programming viewpoint are the on-chip compiler and the UART provided as development software. Architecturally, the speed with which operations can be performed with astute programming on the dual stacks is rewarding. As the software development progresses, one can continually find faster ways to accomplish things that look at first like they are already optimal. I/O on the RTXEB also proved to be trivial

Another feature of the RTX 2001A that was found to be extremely useful in this application was the use of the 32 user memory variables addressed by the User Base Register. Using these variables and addressing them by their literal values cut the execution time of both the filter and the CFT to at least one third of the original time.

Closing the loop in the control of the prototype static inverter represents a significant improvement in this technology. Future developments are now possible in the areas of interactively changing the shape, phase, frequency, amplitude, and harmonic content of the output waveform. Dynamically changing loading conditions are handled extr:mely well within a reasonable range with the controller described in the paper. The five external interrupt lines provided on the RTX $2001 \mathrm{~A}$ would make it easy to impiement any or all of the features described above. It would seem most feasible at this stage that a graphically oriented software package would be developed to communicate with an operator and the external interrupts would change parameters in the control algorithm accordingly.

3.6 Final calculation results for a space-deployed minimum power-to-weight ratio PASC system at a $100 \mathrm{MWe}$ level.

In response to a desire for a determination of the power-to-weight ratio for the PASC technology at a nominal $100 \mathrm{MWe}$ size, the contractor has supplied previously an estimate for this information. The assumptions and details of the calculation were covered in the April 1989 - Sept 1989 Technical Progress Report. In particular, the source MHD system to which it is assumed, the PASC system would be connected is defined as:

Source MHD diagonal terminal voltage at raled current $=1700$ volts dc.

Rated source total diagonal current $=62,700$ amperes $\mathrm{dc}$

Desired PASC secondary voltage $=$ approximately $100 \mathrm{Kv} \mathrm{dc}$.

Additional work in changing the controller to only one or more single board computers (SBC), from the present Feasibility Demonstration system Microvax II with KXT-11 SBC, as discussed above in section 3.5 on the data acquisition and control system, indicates that the power-to-weight conversion ratio can be improved by approximately 200 pounds by the incorporation of such a system. For purposes of completeness we repeat below the basic system design assumptions. 


\subsubsection{Basic Assumptions Used In The Power-to-weight Ratio Calculation}

The value obtained for a weight estimate of the PASC or any technology is highly dependent upon the assumptions made in the design, especially since a full scale detailed system design does not exist. We have assumed the following:

a. There is a reasonable amount of liquid hydrogen around for cooling of the transformer conductors, core, and GTO heat sinks. Since a twoterminal diagonal MHD generator is assumed as a source, and the GTO thyristors can be paralleled together to handle the high primary current of the generator, only two distributed plate heat sinks will be required which may be cooled by liquid hydrogen conduction cooling and circulation. A detailed calculation of how much hydrogen would be gassified in vaporization has not been done but it does not appear to be excessive for a 600 second mission. With this assumption, the resulting design is conservative i.e., the resulting design is a steady state design.

b. It is assumed that the desired output voltage is nominally $100 \mathrm{Kv}$ d.c. We have included the weight of a generic full wave d.c. rectifier following the PASC system in the calculation although ts design will depend somewhat on the required weapons system internal impedance and loading characteristics. However, for simple resistive or leading power factor loads the requirements of such a rectifier will be very simple, requiring no large filtering capacitor, since the PASC system would be programmed in a standard d.c.-to-d.c. square wave switching mode which results inherently in almost no ripple in d.c. output. This is especially true if the design switching frequency is high. Clever heat sink design will be required because of isolation requirements. Based upon a load curient of 1000 d.c. amperes, the total weight for such a rectifier should be less than 500 kilograms.

c. In view of $b$., and because transformer weight reduction results with increasing operational frequency, it is desirable to seek a high frequency design. The upper frequency of operation will be limited either by GTO minimum timing limitations or by the GTO switching transition losses. Because of the increasing relative efficiency of large GTO thyristor units together with assumed high frequency of operation, only a single phase inverter will be necessary, which will limit the gate switching component count.

d. In a d.c.-to-d.c. switched design, such as contemplated here, the control computer speed requirements are very easily realized even up to switching frequencies of $50 \mathrm{KHz}$, because no complex waveform synthesis is required and all of the switching gates are multiplexed in parallel either in a positive or negative polarity grouping. The gate switching interface design must however, be carefully done since relatively large amounts of gate switching power must be accommodated in parallel at high frequencies and even symmetric current loading of the GTO thyristors must be accomplished. 
In the discussions which follow, the design considerations have been divided into two main areas: the design of the PASC switching matrix, and design of the PASC transformer.

The PASC switching matrix is assumed to be on a common ground heat sink located at the exhaust potential of the diagonally connected generator energy source. Careful design must be done to balance symmetrically the large diagonal current between approximately forty 2000 ampere, 4000 volt GTO gates. The design of the PASC transformer, since it is the largest contributor to the PASC system mass, is now considered in detail.

\subsubsection{Design of the PASC Transformer}

The conversion of energy in the PASC transformer will have a significant effect upon the performance and efficiency of the resulting PASC system. There are many interacting design parameters in a transformer and each has an important influence on overall system weight, efficiency and cost. Because of these parameter interdependences, there are many trade-offs to consider in achieving design optimization. Time and funding limitations permit only a first pass estimate of an such a optimized design. Again, the specific details of the calculation are given in referenced report cited previously.

\subsubsection{Final Design Transformer Loss Calculation}

After the selection of Supermendur as the transformer core material of choice, a simple core transformer design was assumed using 0.002 " laminations. As described above, a program to compare Supermendur designs at different frequencies, wire sizes, and dimensions was constructed and run. Because of Supermendur's high saturation point, it can operate at 1.5 Tesla peak flux densities at higher frequencies with small core losses relative to other known materials. Beginning core configurations had lengths of $160^{\prime \prime}$ and 200", for initial primary and secondary wire diameter of $.5 \mathrm{inch}$. Later, secondary wire diameters of 0.75 inch and 1.00 inch were also evaluated. For a fixed width of core of $15 \mathrm{inch}$, core thicknesses of 25,15 , and 5 inches were evaluated. The wire operating temperature was assumed fixed at $173 \mathrm{Kelvin}$, assuming a thermal heat loss temperature differential of approximately 150 Kelvin. All results were calculated using a computer program written in $\mathrm{C}$. Initial evaluations were obtained at $10 \mathrm{KHz}$ without regard for conductor skin effect. After initial evaluations, a final program modification, adding wire skin effect at $10 \mathrm{KHz}$ was made. Individual losses for both primary and secondary were calculated to observe which loss was dominant and to refine the finally selected wire sizes and the balance between core and winding losses.

Analysis of the resulting total losses in the transformer, included both core losses and winding losses as computed by the program leads to the following conclusions:

Because primary current is 60,000 ampere, the initial results were dominated by primary wire loss. Since the major wire losses occur in the primary, the primary conductor was replaced with a fabricated rectangular slab in order to lower its resistance. Larger diameter wire for the secondary was also then selected in order to cut secondary losses. Larger wire size of course increased the required transformer window dimensions and transformer weight, but not very significantly in comparison with the winding loss reduction. Finally, in order to reduce skin effect and guarantee no localized winding hot spots, hollow wire with a duct at the center 
of about $0.3^{\prime \prime}$ to flow pressurized liquid hydrogen cooling was assumed for the secondary winding. From the result of data using 0.5 inch diameter wire, which as stated above was known to be too small, gave total losses were about 2.5 megawatts. Winding losses were lower when primary wire's diameter set to be 1.0 inch. This reduction in wire losses however increased considerably the total weight of the transformer. Initial comparisons for both primary and secondary windings assumed the same diameter wire for all cases. An eventual crosssectional area of 30 square inches reduced the final primary losses so that total losses of about 0.9 megawatt were obtained for the entire transformer. The total t:ansformer weight of this final design increased moderately to $2,386 \mathrm{Kg}$. The resulting design now has core losses of 0.8 megawatt which are higher than copper losses. Again, the objective has been to go for minimum mass: significantly lower losses could be achieved at mass expense by cutting the frequency of operation and working at lower maximum flux density. Under the final design assumptions, the transformer is 62 inches long by 40 inches width and 69" thick, including insulting layer. Wire in primary and secondary are of cross section of 10 square inch and 1 inch diameter respectively.

\subsubsection{Switching Losses and Final Power-to-Weight Ratio Results}

The design of the PASC transformer, using 0.002 " Supermendur core material has been carricd out with the highest priority objective of minimizing the mass of the system. There is a trade-off between mass of the final system and the core dissipation and solid state switching losses because the mass of the transformer can be reduced as the switching frequency is increased while the losses will directly increase with switching frequency. In addition, the resulting desion is a steady state design: the transtormer and all components should be able to last ind efinitely providing the cooling requirements are met. The losses in the GTO and thyristor switches (5.3 MWe), have been estimated based upon a switching frequency of $10 \mathrm{KHz}$ with square waveform generated input for the inverter and a full wave rectified waveform for the $100 \mathrm{KV}$ a.c. square wave output of the transformer. The losses were calculated based upon data sheet information from the manufacturer (TOSHIBA SG2500GXH21 units) of the solid state components. If it is decided that a higher mass can be allocated to the power conversion equipment, a lower value of losses can be generated. Although detailed comparisons have not been done it appears that if a mass twice as great as that given below could be tolerated for the PASC system, the losses could be cut to about $1 / 3$ of the $6.3 \mathrm{MW}$ desion. The final modified design results, including the reduction of 200 pounds by adding the new control and data/acquisition computer system, are as follows:

Total PASC system mass (transformer, switch matrix, computer system, rectifier, switching interface) $=2749 \mathrm{Kg}$.

Total PASC system losses $=6.3 \mathrm{MWe}$ at $100 \mathrm{MWe}, 10 \mathrm{KHz}, 100 \mathrm{KV}$ d.c., $1 \mathrm{~K}$ amps.

Power-to-weight ratio at $100 \mathrm{MWe}$ conversion level $=36.4 \mathrm{KWe} / \mathrm{Kg}$.

$=16.53 \mathrm{KWe} / \mathrm{lb}$. 


\subsection{Final Conclusions and Recummendations}

In summary we draw the following conclusions at the end of this project:

a. The PASC concept feasibility to perform computer controlled pulse formation has been successfully demonstrated for a wide range of symmetric bi-polar pulse waveforms (sinusoidal, square wave, and ramp sawtooth), over frequency ranges varying from 60 to $600 \mathrm{~Hz}$. The demonstration has been achieved by actual experiment on a prototype system with very heavy and strong reliance on backup validated computer simulations of the design and the experimental cases which have been measured. Computer performance simulations have been achieved using standard industry programs SPICE[3] and EMTP[2] along with parallel custom written software routines.

b. Demonstration of the power extraction and consolidation processes has used $16 \mathrm{dc}$ power supplies to crudely represent 16 electrode pairs of a Faraday connected MHD generator source. Computer simulation studies (not part of this contract), of the internal dynaruic interaction expected between the time-dependent gas dynamics of a Faraday configuration MHD duct and the pulsed PASC C/I power extraction process are continuing to determine to what extent the dynamics of the $C / I$ process are limited by the MHD power duct performance.

c. Computer simulation work, which follows very closely the validated simulations already mentioned above, indicates that $\mathrm{C} / \mathrm{I}$ using the PASC process can be successfully done for diagonally connected MHD generators as well as for Faraday connected generators. However, the use of the PASC connection for two-terminal, diagonally connected generators offers only the following significant advantages over competing line commutated bulk power inverters:

c.1 the possibility of control of power factor output without reactive compensation,

c.2 the direct inversion and the ability to operate in program variable pulse mode over wide frequency range,

c.3 the ability to generate high purity single frequency sinusoidal waveforms without any significant filtering.

d. For multiple section, diagonally connected subsection generators, the PASC system could provide program variable local current control balancing in place of alternative current shuffle circuits.

e. Although PASC C/I feasibility has beeen demonstrated experimentally only for (zero dc content), bipolar pulse waveforms using transformers for the consolidation and pulse superposition synthesis process, simulation work has shown that the use of capacitors along with inductors will permit the PASC technique to generate any desired monopolar pulse waveform. For monopolar waveform synthesis it may be possible to substitute natural commutation thyristor gating for symmetric mode GTOs.

f. For space-based power conversion from $1.7 \mathrm{~K}$ vdc to $100 \mathrm{~K}$ vdc at the $100 \mathrm{MW}$ electrical level the power-to-weight conversion ratio has been estimated by calculation to be 36.4 $\mathrm{KWe} / \mathrm{kg}$ for a complete system as defined in section 3.0 . 
g. For arbitrarily shaped bipolar pulse waveforms, symmetric voltage characteristic GTOs must be employed. These GTOs are now commercialiy available in sizes to meet almost any PASC system requirement but are expensive and vendor delivery lead times are long. This is because commercial and consummer applications do not have the stringent space-based requirements on mass and efficiency.

h. Closed-loop control of harmonic generation by PASC systerns has been demonstrated and examined using computer simulation. Many other control strategies are available and need to be examined in the future for this technology.

i. For PASC transformer $\mathrm{C} / \mathrm{I}$ systems the tradeoffs on number of transformers, transformer material and connection configurations, required inductances, losses, and coupling coefficients need significant further study and research, especially relative to a specific design application.

\subsection{References}

[1] Rosa, R. J., Magnetohydrodynamic Energy Conversion, McGraw-Hill, 1968.

[2] Dommel, H. W., "Digital Computer Solution of Electromagnetic Transients in Single and Multiphase Networks," IEEE Transactions on Power Apparatus and Systems, vol. PAS-88, pp 388-399, April 1969.

[3] Nagel, L. W., and Peterson, D. O., "SPICE, simulation programs with integrated circuits analysis," ERI,-M382, Dept. of Electrical Engineering, University of California, Berkeley, 1973.

[4] B. A. Francis and W. M. Wonham, "The Intemal Model Principle of Control Theory", Automatica, Vol. 12, pp. 457-465, 1976.

[5] Gene F. Franklin, J. David Powell, and Abbas Emami-Naeini, Eeedback Control of Dynamic Systems, Reading, Mass.: Addison-Wesley Publishing Co., 1987.

[6] Overview of Dr. C. H. Ting's work o.1 The Continuous Fourier Transform, RTXpress, Vol. 1, No. 3, p. 7, December, 1989. 


\section{Appendix A -. Source Listings}

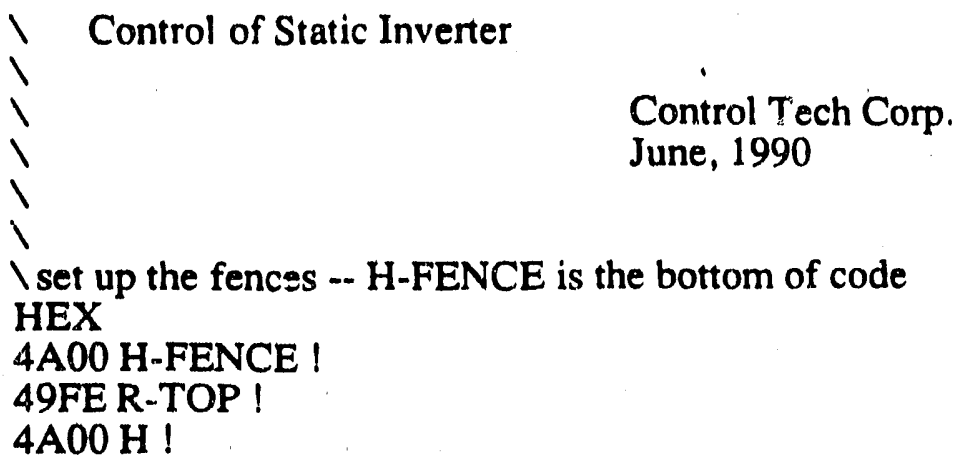

Isame as $\mathrm{C}$, but data goes into user data area -- not code area

:D, 1 ALLOT THERE 1- C! ; I make sure you use D, an even \# of times

\section{DECIMAL}

IVariables

VARIABLE SINTable \allocation for the 200 byte array SINTable

0 D. $1 \mathrm{D}, 2 \mathrm{D}, 3 \mathrm{D}, 4 \mathrm{D}, 5 \mathrm{D}, 6 \mathrm{D}, 7 \mathrm{D}, 8 \mathrm{D}, 9 \mathrm{D}$,

$10 \mathrm{D}, 11 \mathrm{D}, 12 \mathrm{D}, 13 \mathrm{D}, 14 \mathrm{D}, 15 \mathrm{D}, 16 \mathrm{D}, 17 \mathrm{D}, 18 \mathrm{D}, 19 \mathrm{D}$,

$20 \mathrm{D}, 21 \mathrm{D}, 22 \mathrm{D}, 23 \mathrm{D}, 24 \mathrm{D}, 25 \mathrm{D}, 26 \mathrm{D}, 27 \mathrm{D}, 28 \mathrm{D}, 29 \mathrm{D}$,

$30 \mathrm{D}, 31 \mathrm{D}, 32 \mathrm{D}, 33 \mathrm{D}, 34 \mathrm{D}, 35 \mathrm{D}, 36 \mathrm{D}, 37 \mathrm{D}, 38 \mathrm{D}, 39 \mathrm{D}$,

$40 \mathrm{D}, 41 \mathrm{D}, 42 \mathrm{D}, 43 \mathrm{D}, 44 \mathrm{D}, 45 \mathrm{D}, 45 \mathrm{D}, 46 \mathrm{D}, 47 \mathrm{D}, 48 \mathrm{D}$,

$49 \mathrm{D}, 50 \mathrm{D}, 51 \mathrm{D}, 52 \mathrm{D}, 53 \mathrm{D}, 54 \mathrm{D}, 55 \mathrm{D}, 56 \mathrm{D}, 57 \mathrm{D}, 57 \mathrm{D}$,

$58 \mathrm{D}, 59 \mathrm{D}, 60 \mathrm{D}, 61 \mathrm{D}, 62 \mathrm{D}, 63 \mathrm{D}, 64 \mathrm{D}, 65 \mathrm{D}, 65 \mathrm{D}, 66 \mathrm{D}$,

$67 \mathrm{D}, 68 \mathrm{D}, 69 \mathrm{D}, 70 \mathrm{D}, 71 \mathrm{D}, 71 \mathrm{D}, 72 \mathrm{D}, 73 \mathrm{D}, 74 \mathrm{D}, 75 \mathrm{D}$,

$76 \mathrm{D}, 76 \mathrm{D}, 77 \mathrm{D}, 78 \mathrm{D}, 79 \mathrm{D}, 80 \mathrm{D}, 80 \mathrm{D}, 81 \mathrm{D}, 82 \mathrm{D}, 83 \mathrm{D}$,

$83 \mathrm{D}, 84 \mathrm{D}, 85 \mathrm{D}, 86 \mathrm{D}, 86 \mathrm{D}, 87 \mathrm{D}, 88 \mathrm{D}, 89 \mathrm{D}, 89 \mathrm{D}, 90 \mathrm{D}$,

$91 \mathrm{D}, 92 \mathrm{D}, 92 \mathrm{D}, 93 \mathrm{D}, 94 \mathrm{D}, 94 \mathrm{D}, 95 \mathrm{D}, 96 \mathrm{D}, 96 \mathrm{D}, 97 \mathrm{D}$,

$98 \mathrm{D}, 98 \mathrm{D}, 99 \mathrm{D}, 100 \mathrm{D}, 100 \mathrm{D}, 101 \mathrm{D}, 101 \mathrm{D}, 102 \mathrm{D}, 103 \mathrm{D}, 103 \mathrm{D}$,

$104 \mathrm{D}, 104 \mathrm{D}, 105 \mathrm{D}, 106 \mathrm{D}, 106 \mathrm{D}, 107 \mathrm{D}, 107 \mathrm{D}, 108 \mathrm{D}, 108 \mathrm{D}, 109 \mathrm{D}$,

$109 \mathrm{D}, 110 \mathrm{D}, 111 \mathrm{D}, 111 \mathrm{D}, 112 \mathrm{D}, 112 \mathrm{D}, 112 \mathrm{D}, 113 \mathrm{D}, 113 \mathrm{D}, 114 \mathrm{D}$,

$114 \mathrm{D}, 115 \mathrm{D}, 115 \mathrm{D}, 116 \mathrm{D}, 116 \mathrm{D}, 117 \mathrm{D}, 117 \mathrm{D}, 117 \mathrm{D}, 118 \mathrm{D}, 118 \mathrm{D}$,

$119 \mathrm{D}, 119 \mathrm{D}, 119 \mathrm{D}, 120 \mathrm{D}, 120 \mathrm{D}, 120 \mathrm{D}, 121 \mathrm{D}, 121 \mathrm{D}, 121 \mathrm{D}, 122 \mathrm{D}$,

$122 \mathrm{D}, 122 \mathrm{D}, 123 \mathrm{D}, 123 \mathrm{D}, 123 \mathrm{D}, 123 \mathrm{D}, 124 \mathrm{D}, 124 \mathrm{D}, 124 \mathrm{D}, 124 \mathrm{D}$,

125 D, 125 D, 125 D, 125 D, 126 D, 126 D, 126 D, 126 D, 126 D, 126 D,

$127 \mathrm{D}, 127 \mathrm{D}, 127 \mathrm{D}, 127 \mathrm{D}, 127 \mathrm{D}, 127 \mathrm{D}, 127 \mathrm{D}, 127 \mathrm{D}, 127 \mathrm{D}, 127 \mathrm{D}$,

$127 \mathrm{D}, 127 \mathrm{D}, 127 \mathrm{D}, 127 \mathrm{D}, 127 \mathrm{D}, 127 \mathrm{D}, 127 \mathrm{D}, 127 \mathrm{D}, 127 \mathrm{D}, 127 \mathrm{D}$,

HEX

$4500 \mathrm{R}$ !

\CFT Table starts@ @ 4500H

DECIMAL

VARIABLE CFTTable

$\backslash$ allocation for the CFT window

998 ALLOT

HEX

$\checkmark$ Set up the user memory location to point to the first free location.

4440 UBR!

DECIMAL 
IVariable Initialization

1 these are the $\mathbf{3 2}$ allowahle user memory Incation definitions

$00 \mathrm{U!}$

$\checkmark$ sine table pointer -- Reset to use in SINGEN.

$\backslash$ Actual freq is (samp. freq) $*$ Ts $/ \mathrm{T}$.. in this case $(2 \mathrm{kHz}) * 3 / 100=50 \mathrm{~Hz}$.
$31 \mathrm{U} !$
$1002 \mathrm{U}$ !
$\backslash$ Ts

$\backslash 3-8$ are digital filter coefficients

$\backslash 3$
$\backslash 4$
$\backslash 5$
$\backslash 6$
$\backslash 7$
$\backslash 8$
$09 \mathrm{U} !$
$010 \mathrm{U!}$
$011 \mathrm{U!}$
$10012 \mathrm{U!}$
$\backslash 13$
$\backslash 14$
$\backslash 15$
$\backslash 16$
FALSE $19 \mathrm{U!}$
FALSE $20 \mathrm{U!}$
$\backslash 21$
$\backslash$
$\backslash 22-30$
$450030 \mathrm{U!}$
$\backslash 31$

- b0 numerator

- b0 denominator

- b1 num

- bl den

- al num

- al den

$\backslash$ the latest filter output

$\backslash$ intermediate value for filter calculations

$\backslash$ intermediate value for filter calculations

$\backslash$ digital filter gain

- real part of the 3rd harmonic after cft

- the imag part of above

- real part of the 12th harmonic after cft

- the imag part of above

Ia flag indicating that $\mathrm{cft}$ data is valid

$\checkmark$ a flag indicating an overflow in the cft

- difference between most recent a/d value

- and the most distant $\mathrm{a} / \mathrm{d}$ value for $\mathrm{cft}$

- not used

$\checkmark$ cft table index pointer

- count delay while waiting for valid cft data

$\backslash$ CFT constants are arguments for 3 rd and 12 th harmonics
30 CONSTANT 3RDarg
116 CONSTANT 12THarg.
$\backslash 800371000 *$ I
$\backslash 8001451000 * /$

: $\operatorname{SNN}(\mathrm{n} 1--\mathrm{n} 2)$

$\backslash \mathrm{n} 2$ is the $\sin$ of $\mathrm{n} 1$.

$\ln 2$ is between -128 and 128 which represents -1 to 1 .

I SINTable is a table holding 200 points of the first quarter of the sin wave.

796 MOD

DUP $199<$ IF

SINTable+C@ELSE

$\backslash$ Fold over so it is between 0 and 796.

DUP $398<$ IF

398 SWAP. SINTable + C@ ELSE

DUP $597<$ IF

396-SINTable+C@ NEGATE ELSE

DUP $796<$ IF

795 SWAP-SINTable + C@ NEGATE

THEN THEN THEN THEN

;

$\backslash$ end of SIN

$\backslash$ get SINTable(n+1)

\get SINTable(399-n)

$\backslash$ get -SINTable(n-397)

$\backslash$ get -SINTable(796-: ı) 


\section{: SINGEN $(-n)$}

$\backslash$ Places the next value of the sin wave on the stack.

$\backslash$ SINIndex is used to store the position of the sin wave (it is incremented).

$\backslash \mathrm{Ts}$ (samp. per.) and $\mathrm{T}$ ( sin per.) are used to scale the sin wave.

0U@

2U@MOD

DUP $1+0$ U!

1U@796*2U@*/ In2*1s*796/T -. 1 is Ts

SIN

;

lend of SINGEN

HEX

: OUTWORD ( n1 -- )

Staircase limit $\mathrm{nl}$, and send the switch word to the appropriate

$\backslash$ ASIC bus address.

DUP -FF > IF

FFFF $1 A$ G!

DUP EF1 > IF 0000 ELSE

DUP CF3 > IF 0080 ELSE

DUP AF5 > IF 0060 ELSE

DUP 8F7 > IF 001C ELSE

DUP 6F9 > IF 00C3 ELSE

DUP 4FB > IF 003E ELSE

DUP 2FD $>$ IF OOF9 ELSE

DUP FF $>$ IF OOF7 ELSE OOFF

$\backslash$ SINIndex is 0

$\checkmark$ wrap the index around --2 is $T$ \level 8

\level 7

\level 6

$\backslash$ level 5

\level 4

$\backslash$ level 3

$\backslash$ level 2

$\backslash$ level 1

\level 0

THEN THEN THEN THEN THEN THEN THEN THEN

SWAP DROP $19 \mathrm{G}$ !

iPositive ASIC bus address

THEN

DUP -FF $<=$ IF

FFFF $19 \mathrm{G}$ !

DUP -2FD > IF 00F7 ELSE

DUP - 4FB > IF

OOF9 ELSE

DUP -6F9 > IF

003E ELSE

DUP -8F7 > IF 00C3 ELSE

DUP - AF5 > IF

001C ELSE

\level -1

\level -2

\level -3

\level -4

\level -5 


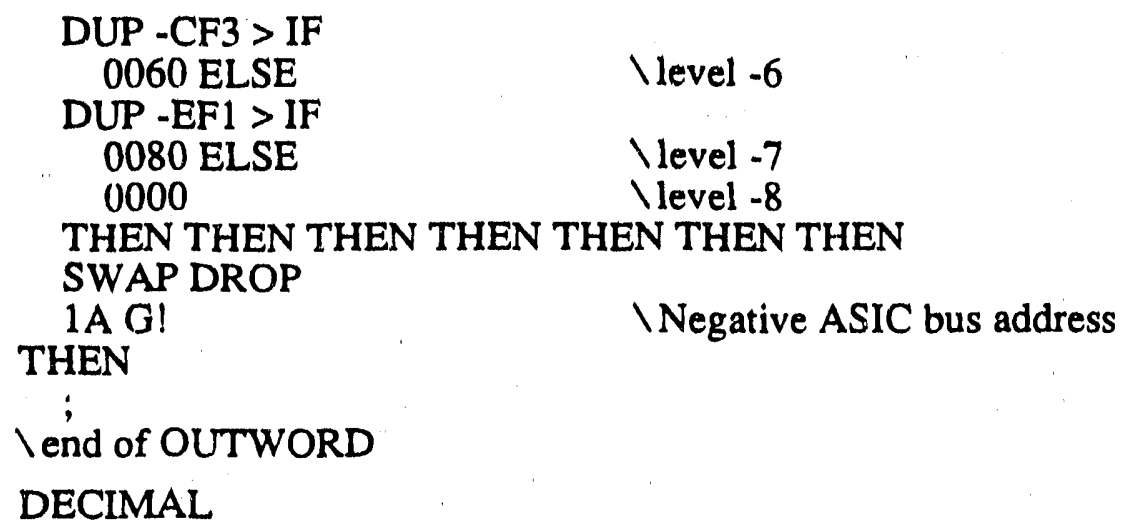

\end of FILTER

: SETFILTCOEFFS (-- )

$\checkmark$ Set up the controller filter, $C(z)$, coefficients: b0num, b0den, b1num, b1den,

$\backslash$ alnum, and alden.

$\backslash$ Actual reference frequency is (samp. freq) $* \mathrm{Ts} / \mathrm{T}$

$|\mathrm{a}|=-2 * \cos (\mathrm{w} 0 * \mathrm{Ts})$

$\backslash \mathrm{a} 2=1$

$\backslash \mathrm{b} 0=\mathrm{k} 2$

$\backslash \mathrm{b} 1=-\mathrm{k} 2 * \cos (\mathrm{w} 0 * \mathrm{Ts})$

$\backslash \mathrm{k} 2$ is scaled by 100

12 U@ DUP 3 U! 1004 U! ( k2 ... )

$997610000 * / 5 \mathrm{U!} 16 \mathrm{U}$ ! (-) \blnum and biden

$199527 \mathrm{U!} 100008 \mathrm{U} ! \quad$ ( - ) \alnum and alden

$\backslash$ bOnum and b0den

; ind of SETFILTCOEFFS

HEX

$\checkmark$ Power down and quit if keystroke is caught

: STOPWAVE (--)

FFFF 001F G!

$\backslash$ Send open signals 
lend of STOPWAVE

DECIMAL

: CLOSEDLOOP ( -- )

$\backslash$ Open loop sine wave generator.

CR@UBR@

17472 UBR!

Save the Configuration Reg and UBR

$\backslash$ set UBR to $4540 \mathrm{H}$

SINGEN

INext sine wave point.

28 G@

255 AND

DUP30 U@ C!

$\backslash$ Get $A / D$ value and start next conversion.

$\backslash$ Look at least significant 8 bits.

30 U@ 1+DUP 18663 > IF DROP 17664 THEN \ check for table wrap

$30 \mathrm{U!}$

128 -

$\backslash$ increment the table index $17920=4600 \mathrm{H}$

$\backslash$ Center it at zero.

$-$

30 *

$\backslash$ Find the difference.

$\backslash$ For numerical scaling.

FILTER

$\backslash$ Apply the controller filter $G(z)$.

OUTWORD \Staircase limit the output, and send it to t': ASIC bus.

TRUE 19 U!

$\backslash$ ok to do an $\mathrm{fft}$ calc

UBR! CR!

$\backslash$ Restore the Configuration Reg and UBR

;

lend of CLOSEDLOOP

\section{: CFT ( -- )}

BEGIN

$\backslash$ stop and open gto's on keystroke

KEY? IF TIMER2 MASK STOPWAVE QUIT THEN

$\backslash y \operatorname{diff}=y(k)-y(k-N)$ where $N$ is window length
30U@C@
$\backslash 30$ is addr of most recent $y$
1 center it $-\mathrm{a} / \mathrm{d}$ is $0-5$ volts

128 -

30 U@ 1+DUP $18663>$ IF DROP 17664 THEN \check for table wrap

C@

128 -

$\backslash 301+$ is addr of oldest $y$

$-21 \mathrm{U!}$

lcenter it

$\backslash$ get last step index

14 U@ DUP $1+500$ MOD $14 \mathrm{U!}$ ! step -- )
। 3 arg corresponds to $-2^{*} \mathrm{pi}^{*} \mathrm{i}^{*} \mathrm{k}^{*} \mathrm{n} / \mathrm{N}$, the argument of the CFT exponential

DUP 3RDarg 796 */MOD DROP

DUP SIN

$\backslash$ the imaginary part of the exponential

21U@*16U@+DUP16U!

DUP ABS $180>$ IF TRUE 20 U! THEN

DUP *

SWAP $199+796$ MOD SIN （ step imag^2 cos -- )

$\backslash$ the real part of the exponential

21U@*15 U@+DUP15 U!

DUP ABS $180>$ IF TRUE 20 U! THEN

DUP *

$\backslash$ the magnitude of the exponential

+ SQRT 100 *

( step 3arg -- )

( step 3arg sin -- )

( step 3arg imag -- )

I check for overflow

( step $3 \arg \operatorname{imag}^{\wedge} 2$-- )
( step imag^2 real -- )

$\checkmark$ check for overflow

( step imag^2 real^$^{\wedge} 2$-- )

( step $|3 r d|--$ ) 
Istart on 12th harmonic

112 arg is same as 3 arg but for 12 harmonic

SWAP 12THarg $796 * / M O D$ DROP

DUP SIN

The imaginary part of the exponential

21U@*18U@+DUP18 U!

DUP ABS 180 > IF TRUE 20 U! THEN

DUP *

SWAP $199+796$ MOD SIN $\quad\left(13 \mathrm{rdl} \mathrm{imag}^{\wedge} 2 \cos --\right)$

$\backslash$ the real part of the exponential

21U@*17U@+DUP17 U!

DUP ABS $180>$ IF TRUE 20 U! THEN

DUP *

$\backslash$ the magnitude of the exponential

+ SQRT

$\backslash$ take ratio and adjust controller gain

/ DUP
$\backslash$ this is a delay to wait for valid data -- also dont do another point

( 13 rdl 12arg $\cdots$ )

( |3rdl 12arg sin -- )

( |3rdl 12arg imag -- )

$\backslash$ check for overflow

( |3rdl '2arg imag^2 -- )

(13rdlimag 2 cos - )

1 until an interrupt has occured -- 19 is set true in interrupt

31 U@ DUP 32000 < IF 1+ 31 U! FALSE 19 U! ELSE DROP THEN

$\checkmark$ a true flag means data valid

19U@

DUP ROT

( $13 \mathrm{rdl}$ imag^2 real -- )

(check for overflow

( $\mid 3 \mathrm{rdl} \operatorname{imag}^{\wedge} 2 \mathrm{real}^{\wedge} 2$-- )

AND $110>$ IF 12 U@ 1+ 12 U! SETFILTCOEFFS THEN ( ratio flag -- )

90 AND < IF 12 U@ 1-12 U! SETFILTCOEFFS THEN (-- )

$\backslash$ if there was an overflow, start over with new data

20 U@ UNTIL FALSE 20 U!

;

: STARTCLOSED ( -- )

Start the open loop driver by enabling the timer 0 interrupt which runs

$\backslash$ CLOSEDLOOP at each interrupt.

SETFILTCOEFFS

$4000 \mathrm{TC} 2$ !

['] CLOSEDLOOP 9 !INTERRUPT

TIMER2 UNMASK

BEGIN

$031 \mathrm{U}$ !

CFT

AGAIN

;

lend of STARTCLOSED

\a flag to indicate valid data in the cft table 

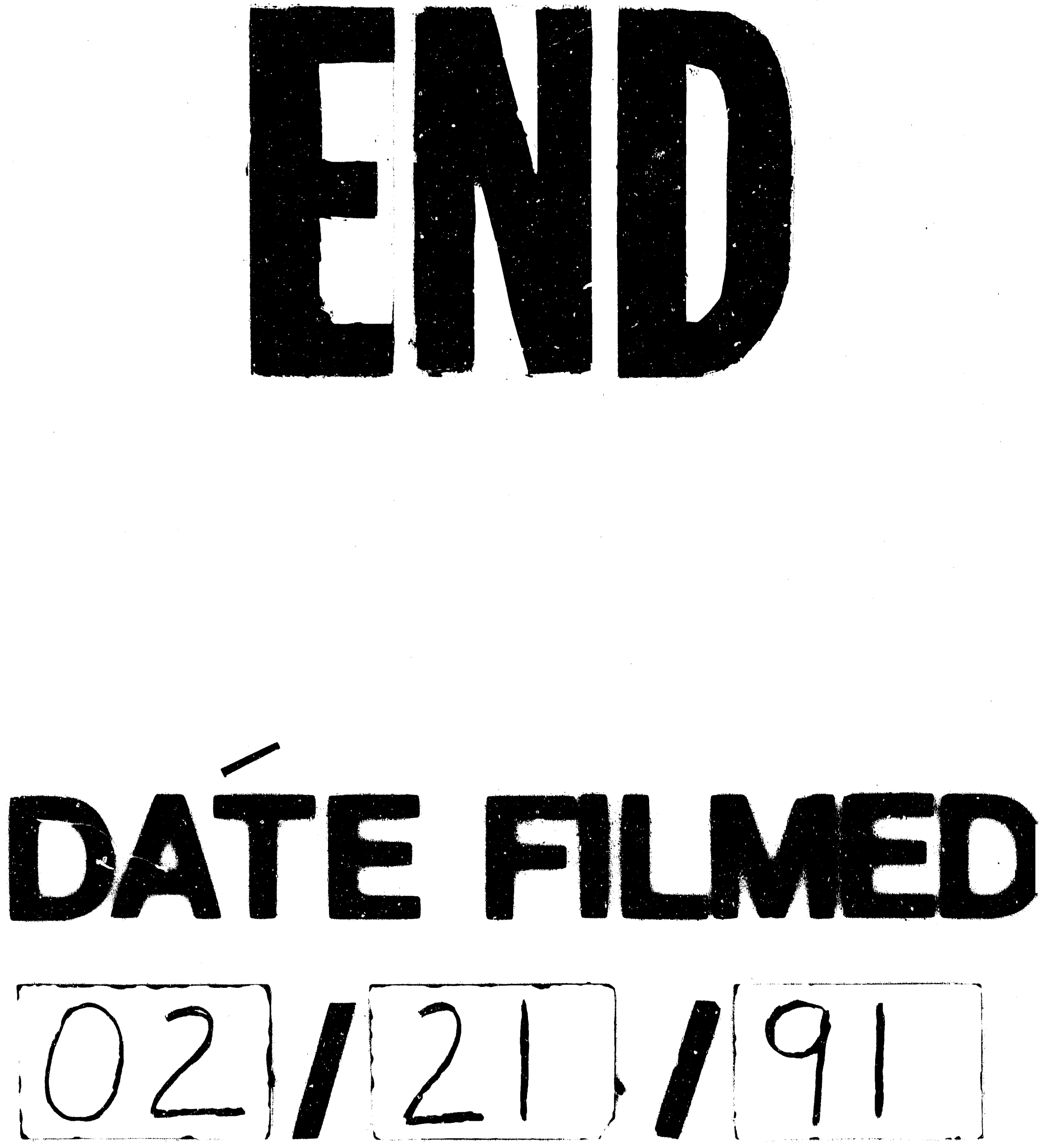
